TOKYO J. MATH.

VOL. 6, No. 2, 1983

\title{
Fonctionnelles Analytiques a Porteur Non Borné sur $C$
}

\author{
A. MERIL \\ Université de Bordeaux \\ (Communicated by M. Morimoto)
}

\section{Résumé}

Nous montrons que le dual d'un espace de germes de fonctions holomorphes au voisinage d'un convexe fermé à croissance contrôlée à l'infini est isomorphe à un espace de fonctions holomorphes de type exponentiel dans un cône $\Gamma$. Notre preuve est explicite.

\section{Introduction}

Soit $\Omega$ un convexe fermé non compact de $C^{n}$; on montre aisément que $\Omega$ est déterminé de façon biunivoque par un cône convexe $\Gamma$ (relativement) ouvert, de sommet l'origine et une fonction d'appui $a$ sur $\Gamma$.

Il existe deux espaces différents de germes de fonctions holomorphes au voisinage de $\Omega$ et à croissance contrôlée à l'infini; l'un noté $\mathscr{H}(\Omega)$ est constitué de germes de fonctions holomorphes sur des $\varepsilon$-voisinages et l'autre $\mathscr{H}_{0}(\Omega)$ s'obtient en utilisant des voisinages "coniques". Lorsque $\Omega$ ne contient pas de droites réelles, ces espaces sont isomorphes à des espaces de fonctions holomorphes dans le cône $\Gamma$ et de type exponentiel $a$.

Ces espaces furent introduits dans des cas très particuliers par T. Kawaï [6]. Dans le cas général, l'étude fut faite par J. W. de Roever qui prouve les isomorphismes par des méthodes de $L^{2}$-estimations et en étendant le principe fondamental de Ehrenpreis-Palamadov. Ce théorème d'isomorphisme fut retrouvé dans le cas des $\varepsilon$-voisinages et de manière indépendante par M. Morimoto [11] lorsque $n=1$ et $\Omega$ est une demi-bande. Il apparait (voir [1]) que la $L^{2}$-estimation n'est pas élémentaire, même pour $n=1$. Il nous paraît donc naturel et intéressant de refaire dans le cas général une preuve analogue à celle de M. Morimoto en utilisant (pour $n=1$ ) les transformées de Laplace et de Cauchy, en espérant dans le cas 
général (i.e. $n \geqq 2$ ) réobtenir les résultats de J. W. Roever dans l'optique de Martineau-Aizenberg. Certains résultats furent obtenus dans cette voie (dans le cas d'un produit de demi-bandes) par Morimoto-Sargos [12], il est clair, à la lumière de ce travail, que nos résultats peuvent immédiatement être étendus au cas où $\Omega$ convexe fermé non borné de $C^{n}$ est un produit cartésien de $n$ convexes fermés non compacts de $C$.

L'utilité de ces espaces de fonctionnelles analytiques à porteur non borné est multiple. Selon J. W. de Roever [15] chapitre 1, ils sont d'un certain intérêt en mécanique quantique des champs. On peut aussi y étudier des équations de convolution, généralisant des équations différences différentielles pour cela, voir Berenstein-Struppa [2] et Méril [8], [9], [10].

Récemment, la méthode que nous utilisons fut retrouvée de manière indépendante par B. Malgrange [7] dans l'étude des microfonctions. Les espaces sur lesquels il travaille sont assez voisins du nôtre.

Nous tenons à remercier le Professeur R. Gay pour les conseils qui nous furent donnés et nous croyons que sans son aide cet article n'aurait pas vu le jour.

\section{Chapitre 0}

QUELQUES RAPPELS SUR LES E.L.C. ET LA CONVEXITE

On désigne par $\boldsymbol{N}$ (resp. $\boldsymbol{R}, \boldsymbol{C}$ ) l'ensemble des entiers naturels (resp. le corps des nombres réels, le corps des nombres complexes).

Si $\alpha \in N^{n}$ est un multi-indice, on note par $|\alpha|$ sa longueur, $|\alpha|=$ $\alpha_{1}+\cdots+\alpha_{n}$. L'opérateur de dérivation:

$$
\frac{\partial^{|\alpha|}}{\partial x_{1}^{\alpha_{1}} \cdots \partial x_{n}^{\alpha_{n}}}
$$

opérant sur les fonctions de la variable $x \in R^{n}$ sera noté $D_{x}^{\alpha}$.

$\mathrm{Si} V$ est un sous-ensemble d'un espace topologique $X$ on notera $\bar{V}$ son adhérence, $\partial V$ sa frontière et $V^{0}$ son complémentaire. On note par $\langle y, \xi\rangle$ le produit scalaire $\sum_{j=1}^{n} y_{j} \xi_{j}$ des vecteurs $y=\left(y_{1}, \cdots, y_{n}\right)$ et $\xi=$ $\left(\xi_{1}, \cdots, \xi_{n}\right)$ de $\boldsymbol{R}^{n}$.

\section{§1. E.L.C..}

Dans la suite le sigle E.L.C. désignera un espace vectoriel topologique localement convexe séparé.

DEFinition 0.1.1. Soient $X, Y$ deux E.L.C. et $u$ une application linéaire de $X$ dans $Y$. L'application $u$ sera dite compacte (resp. faible- 
ment compacte) s'il existe un voisinage de zéro $V$ dans $X$ tel que $u(V)$ soit relativement compact (resp. relativement faiblement compact) dans $Y$.

DEFINITION 0.1.2 ([4]). Soit $\left(E_{n}, \pi_{n, p}\right)\left((n, p) \in N^{2}\right)$ un système projectif d'E.L.C.. On dit que la limite projective topologique $E=\lim _{n} E_{n}$ est un espace du type F.S si les applications linéaires $\pi_{n, p}$ sont compactes (resp. faiblement compactes).

DEFinition 0.1.3 ([4]). Soit $\left(F_{n}, \pi_{n, p}^{\prime}\right)\left((n, p) \in N^{2}\right)$ un système inductif d'E.L.C. tel que les applications $\pi_{n, p}^{\prime}$ soient injectives. On dit que la limite inductive topologique $F=\lim _{n} F_{n}$ est un espace du type D.F.S si les applications linéaires $\pi_{n, p}^{\prime}$ sont compactes.

Proposition 0.1.4 ([4]). Soit $E=\lim _{n} E_{n}$ un espace du type F.S. Si pour tout $n$ et tout $m$ tels que $m>n \pi_{m, n} E_{m}$ est dense dans $E_{n}$, le dual topologique $E^{\prime}$ de $E$, qui s'identifie à $\lim _{n} E_{n}^{\prime}$, est un espace du type D.F.S.. Soit $F=\lim _{n} F_{n}$ un espace du type D.F.S.. Le dual topologique $F^{\prime}$ de $F$ qui s'identifie $\grave{a} \lim _{n} F^{\prime}$ est un espace du type F.S.

Pour d'autres propriétés de ces espaces, nous renvoyons à [4] et [13] (par exemple).

\section{§2. Quelques exemples.}

Soit $\mathcal{O}$ un ouvert non vide de $\boldsymbol{R}^{p}$. Soit $m \in N$. Une fonction continue et positive $M$ sur $\mathscr{O}$ sera dite un poids. On désignera par $\mathscr{C}^{m}(\mathcal{O}, M)$ l'espace des fonctions continues $\Phi$ de classe $\mathscr{C}^{m}$ sur $\mathscr{O}$ telles que:

$$
\|\Phi\|_{m}=\sup _{\substack{x \in \Phi^{\infty} \\|\alpha| \leq m}}\left|D^{\alpha} \Phi(x)\right| M(x)<+\infty .
$$

On suppose que $\mathcal{O}$ est réunion d'une suite croissante d'ouverts $\left(\mathcal{O}_{m}\right)_{m \in N}$. Soit $M=\left(M_{m}\right)_{m \in N}$ une famille de poids telle que $M_{m}$ soit défini sur $\mathscr{O}_{m}$ et que $M_{m+1}(x) \geqq M_{m}(x)$ pour $x \in \mathcal{O}_{m}$. On définit l'espace $\mathscr{C}^{\infty}(\mathscr{O}, M)$ comme étant égal à $\lim _{m} \mathscr{C}^{m}\left(\mathcal{O}_{m}, M_{m}\right)$.

Proposition 0.2.1. Avec les données ci-dessus s'il existe une exhaustion $\left(K_{k, m}\right)_{k} \in N$ de $\mathcal{O}_{m}$ pour tout $m$ par des ouverts relativement compacts tels que $\bar{K}_{k, m} \subset K_{k+1, m} \subset \mathcal{O}_{m}$ et tels que, pour tout $\varepsilon>0$, il existe $l(\varepsilon) \in N$ pour lequel $x \in \mathcal{O}_{m} \backslash K_{m, k(s)}$ implique $M_{m}(x) \leqq \varepsilon M_{m+1}(x)$, alors l'espace $\mathscr{C}^{\infty}(\mathcal{O}, M)$ est $d u$ type F.S. 
Preuve ([13]). L'inégalité des accroissements finis prouve que l'application de restriction

$$
\pi_{m+1, m}: \mathscr{C}^{m+1}\left(\mathcal{O}_{m+1}, M_{m+1}\right) \longrightarrow \mathscr{C}^{m}\left(\mathcal{O}_{m}, M_{m}\right)
$$

est compacte.

Definition 0.2.2. Soient $\Omega$ un ouvert de $C^{n}$ et $M$ un poids sur $\Omega$. On désigne par $\mathscr{C}(\Omega, M)$ le $C$-espace vectoriel:

$$
\mathscr{C}(\Omega, M)=\left\{f \in \mathscr{C}(\Omega)\left|\|f\|=\sup _{\boldsymbol{z} \in \boldsymbol{Q}}\right| f(z) \mid M(z)<+\infty\right\}
$$

où $\mathscr{H}(\Omega)$ désigne l'espace de toutes les fonctions holomorphes dans $\Omega$.

Nous rappelons deux conditions techniques classiques ([13], [15]) dont la fonction est d'assurer que certains espaces sont nucléaires.

Soit $\left(\Omega_{m}\right)_{m \in N}$ une suite croissante (resp. décroissante) de domaines de $C^{n}$ de réunion (resp. d'intersection) $\Omega$. Soit $M=\left(M_{m}\right)_{m \in N}$ une suite de poids tels que $M_{m}$ soit défini sur $\Omega_{m}$ et $M_{m}(x) \leqq M_{m+1}(x)$ pour $x \in \Omega_{m}$ (resp. $M_{m}(x) \geqq M_{m+1}(x)$ pour $x \in \Omega_{m+1}$ ).

Condition 0.2.3. $\quad H . S_{1}$

Pour tout $m \in N$ il existe $p>m$ (resp. pour tout $p \in N$ il existe $m>p$ ) tel que

$$
\int_{\Omega_{m}}\left(\frac{M_{m}(z)}{M_{p}(z)}\right)^{2} d \lambda(z)<+\infty .
$$

Condition 0.2.4. H.S

Pour tout $m \in N$ il existe $p>m$ (resp. pour tout $p \in N$ il existe $m>p$ ) tel que $\Omega_{m}$ soit recouvert dans $\Omega_{p}$ par des polydisques:

$$
\Delta\left(z, d_{z}\right)=\left\{w \in C^{n}|| z_{j}-w_{j} \mid<d_{z} ; 1 \leqq j \leqq n\right\}
$$

avec

$$
\sup _{\substack{z \in \rho_{m} \\ w \in \Delta\left(z, d_{z}\right) \subset \Omega_{p}}} d_{z}^{-n} M_{p}^{-1}(w) M_{m}(z)<+\infty
$$

Ces conditions assurent que:

Proposition 0.2.5 ([13]). Si les conditions $H . S_{1}$ et H.S $S_{2}$ sont vérifiées, l'espace $\lim _{m} \mathscr{\mathscr { C }}\left(\Omega_{m}, M_{m}\right)$ (resp. $\lim _{m} \mathscr{\mathscr { C }}\left(\Omega_{m}, M_{m}\right)$ ) est un espace du type F.N (resp. D.F.N).

Pour les espaces de fonctions holomorphes du type $\mathscr{H}(\Omega, M)$ il existe un analogue de 0.2.1. 
Proposition 0.2.6. Soit $M_{i}$ un poids sur $\Omega_{i}(i=1,2)$ ouvert de $C^{n}$ tels que $\Omega_{2} \subset \Omega_{1}$ et $M_{1} \geqq M_{2}$ sur $\Omega_{2}$. L'injection de $\mathscr{H}\left(\Omega_{1}, M_{1}\right)$ dans $\mathscr{H}\left(\Omega_{2}, M_{2}\right)$ est continue.

Si $\Omega_{2}=\bigcup_{k} S_{k}$ où $S_{k}$ est un ouvert de $C^{n}$ relativement compact dans $S_{k+1}$ pour tout $k$ et si, pour tout $\varepsilon>0$, il existe $k(\varepsilon)$, tel que $z \in \Omega_{2} \mid S_{k}(\varepsilon)$ implique $M_{2}(z) \leqq \varepsilon M_{1}(z)$, alors l'injection de $\mathscr{\mathscr { C }}\left(\Omega_{1}, M_{1}\right)$ dans $\mathscr{\mathcal { P }}\left(\Omega_{2}, M_{2}\right)$ est compacte. Cette affirmation est encore vraie si on suppose seulement que $\bar{S}_{k}$ est compact dans $\Omega_{1}$.

Preuve. Cf. [13].

\section{§3. Cône convexe dans $\boldsymbol{R}^{n}$.}

Dans toute la suite les cônes considérés seront convexes et de sommet 0 sauf mention expresse du contraire.

Si $\Gamma$ est un cône de $\boldsymbol{R}^{n}$ et $\boldsymbol{S}^{n-1}$ est la sphère unité de $\boldsymbol{R}^{n}$, on désigne par projection de $\Gamma$ sur $S^{n-1}$ et on note $\operatorname{pr}(\Gamma)$ l'ensemble $\Gamma \cap S^{n-1}$. Un sous-cône $\Gamma^{\prime}$ de $\Gamma$ est dit un sous-cône relativement compact du cône ouvert $\Gamma$ si l'ensemble $\operatorname{pr}\left(\Gamma^{\prime}\right)$ est relativement compact dans l'ouvert $\operatorname{pr}(\Gamma)$ de $S^{n-1}$. On notera ceci par $\Gamma^{\prime} \subset \subset \Gamma$.

Soit $\Gamma$ un cône ouvert convexe. Une exhaustion de $\Gamma$ sera la donnée d'une famille $\left(\Gamma_{k}\right)_{k \geqq 1}$ de sous-cônes ouverts et convexes tels que, pour tout $k$,

$$
\begin{aligned}
& \Gamma_{k} \subset \subset \Gamma_{k+1} \subset \subset \Gamma \\
& \Gamma=\bigcup_{k \geq 1} \Gamma_{k} .
\end{aligned}
$$

Une fonction $a$ définie dans $\Gamma$, à valeurs réelles, convexe et positivement homogène d'ordre un sur $\Gamma$ sera dite une fonction d'appui.

Soit $a_{1}$ une fonction d'appui définie sur le cône ouvert convexe $\Gamma_{1}$. On désigne par $\Omega_{1}\left(a_{1}, \Gamma_{1}\right)$ l'ensemble convexe fermé dans $\boldsymbol{R}^{n}$

$$
\text { - } \Omega_{1}\left(a_{1}, \Gamma_{1}\right)=\left\{\xi \in R^{n} \mid-\langle y, \xi\rangle \leqq a_{1}(y) ; \forall y \in \Gamma_{1}\right\} .
$$

Réciproquement, soit $\Omega$ un convexe fermé non vide de $\boldsymbol{R}^{n}$ que l'on représentera comme l'intersection des demi-espaces fermés $H$ de la famille $\mathscr{K}$. des demi-espaces fermés $H \supset \Omega$.

Pour $H \in \mathscr{K}$, soit $y$ l'unique vecteur unitaire "sortant" orthogonal à $\partial H$ pour lequel

$$
H=H_{y}(a)=\left\{\xi \in R^{n} \mid-\langle y, \xi\rangle \leqq a\right\}
$$

où $a \in \boldsymbol{R}$. 
Les vecteurs $y$ ci-dessus varient dans un sous-ensemble de $S^{n-1}$ que nous noterons $\operatorname{pr}\left(\Gamma_{1}\right)$ et qui est la projection du cône $\Gamma_{1}$ qu'il engendre. On montre ([14]) que $\Gamma_{1}$ est un cône convexe (non nécessairement ouvert).

On détermine une fonction $y \rightarrow \widetilde{a}_{1}(y)$ sur $\operatorname{pr}\left(\Gamma_{1}\right)$ par la condition:

$$
\Omega=\left\{\xi \in R^{n} \mid-\langle y, \xi\rangle \leqq \widetilde{a}_{1}\left(y\|y\|^{-1}\right)\|y\|, \forall y \in \Gamma_{1}\right\}
$$

de telle sorte que, pour tout $y \in \Gamma_{1}$

$$
a_{1}(y)=\|y\| \tilde{a}_{1}\left(y\|y\|^{-1}\right)=\sup _{\xi \in \Phi}-\langle y, \xi\rangle
$$

soit la fonction d'appui de $\Omega$. On vérifie que la fonction $a_{1}$ est convexe done continue sur $\Gamma_{1}$.

Il se peut que $\Gamma_{1}$ ne soit pas ouvert dans $R^{n}$ mais il existe $p \leqq n$ tel que $\Gamma_{1}$ soit contenu dans un sous-espace vectoriel de dimension $p$ dans lequel son intérieur "relatif" int $\left(\Gamma_{1}\right)$ soit non vide.

On constate alors que $\Omega=\Omega_{1}\left(a_{1}\right.$, int $\left.\left(\Gamma_{1}\right)\right)$. On obtient ainsi le

THEORÈME 0.3.1 ([14]). Tout convexe fermé $\Omega$ de $\boldsymbol{R}^{n}$ détermine un cône convexe $\Gamma_{1}$ (ouvert dans un sous-espace vectoriel convenable de $\boldsymbol{R}^{n}$ ) et une founction d'appui $a_{1}$ définie sur $\Gamma_{1}$ tels que $\Omega=\Omega_{1}\left(a_{1}, \Gamma_{1}\right)$. Réciproquement chaque cône ouvert convexe $\Gamma_{1}$ et chaque fonction d'appui $a_{1}$ définie sur $\Gamma_{1}$ détermine un convexe fermé. Le cône $\Gamma_{1}$ est ouvert dans $R^{n}$ si, et seulement si, $\Omega_{1}$ ne contient pas de droite.

Dans ces conditions nous dirons que $\Omega_{1}$ et $\left(\Gamma_{1}, a_{1}\right)$ sont en dualité.

Dans la suite, le plus souvent, $n$ sera égal à 2 et $R^{2}$ sera identifié à $C$; le produit scalaire dans $R^{2}=C$ s'exprimant par:

$$
\langle z, \xi\rangle=\operatorname{Re}(z \bar{\xi}) \text {. }
$$

Alors

$$
\Omega=\Omega_{1}\left(a_{1}, \Gamma_{1}\right)=\left\{z \in C \mid-\operatorname{Re} z \bar{\xi} \leqq a_{1}(\xi) ; \forall \xi \in \operatorname{pr}\left(\Gamma_{1}\right)\right\} .
$$

On supposera que $\Omega_{1}\left(a_{1}, \Gamma_{1}\right)$ ne contient pas de droite et que cet ensemble n'est pas compact.

Soient $\Omega$ un convexe fermé et un secteur $\left(\Gamma_{1}, a_{1}\right)$ en dualité. On considère le secteur $\Gamma$ symétrique de $\Gamma_{1}$ par rapport à la première bissectrice

$$
\Gamma=\left\{i \bar{\xi} ; \xi \in \Gamma_{1}\right\}
$$

et on définit sur $\Gamma$ la fonction $a(\xi)=a_{1}(i \bar{\xi})$ qui est évidemment une 
fonction d'appui sur $\Gamma$. En tenant compte de la relation $\operatorname{Re}(i z \xi)=-\operatorname{Im} z \xi$ $(z, \xi \in C)$ on a alors:

$$
\Omega=\Omega_{1}\left(a_{1}, \Gamma_{1}\right)=\{z \in C \mid-\operatorname{Im}(z \xi) \leqq a(\xi) ; \forall \xi \in \Gamma\} .
$$

On conviendra de noter $\Omega \operatorname{par} \Omega(a, \Gamma)$ et on dira encore que $\Omega$ et $(\Gamma, a)$ sont en dualité.

Pour $\Gamma$ et $a$ donnés comme ci-dessus et pour $\xi \in \operatorname{pr}(\Gamma)$ on pose:

$$
H_{\xi}=\{z \in C \mid-\operatorname{Im}(z \xi) \leqq a(\xi)\} .
$$

Si $\xi=e^{i \beta} \in \operatorname{pr}(\Gamma)$ tout $z \in C$ s'écrit de façon unique $z=\lambda e^{-i \beta}+$ $\mu e^{i(\pi / 2-\beta)}(\lambda, \mu \in \boldsymbol{R})$ et $z \in H_{\xi}$ si, et seulement si, $\mu \geqq-a(\xi)$. On a:

$$
\Omega=\Omega(a, \Gamma)=\bigcap_{\xi \in \mathrm{pr}(r)} H_{\xi} .
$$

Pour $\varepsilon>0$ l' $\varepsilon$-voisinage $\Omega_{\varepsilon}$ de $\Omega$ peut s'écrire:

$$
\Omega_{\varepsilon}=\Omega(a+\varepsilon|\xi|, \Gamma) \text {. }
$$

Pour $\xi \in \operatorname{pr}(\Gamma)$ le demi-plan fermé:

$$
H_{\varepsilon, \varepsilon}=\{z \in C \mid \operatorname{Im}(z \xi)+a(\xi) \leqq-\varepsilon\}
$$

est le demi-plan fermé "d'appui" à $\Omega_{s}$ ne contenant pas $\Omega_{s}$, tel que sa frontière $\Delta_{\xi, \varepsilon}$ soit une droite de vecteur directeur $\bar{\xi}$.

Soient maintenant $\xi_{1}$ et $\xi_{2}$ deux éléments de $\operatorname{pr}(\Gamma)$ et $\Gamma^{\prime}$ le secteur convexe engendré par $\xi_{1}$ et $\xi_{2}$. Pour $\xi \in \operatorname{pr}\left(\Gamma^{\prime}\right)$ on a

$$
H_{\xi_{1}, \varepsilon} \cap H_{\xi_{2}, \varepsilon} \subset H_{\xi, \varepsilon} \text {. }
$$

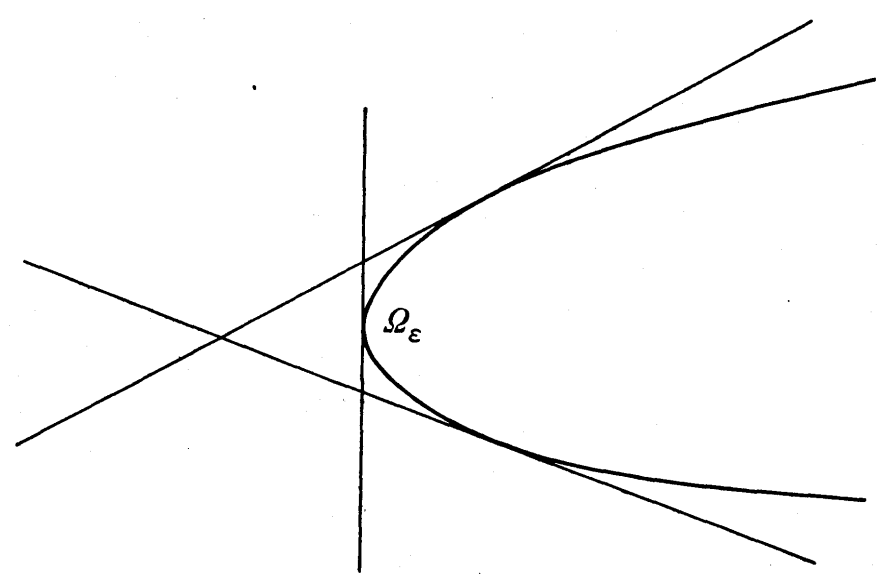


A. MERIL

On remarquera surtout que l'intersection d'une droite parallèle à $\Delta_{\xi, \mathrm{s}}$ avec $\Omega_{c}$ (ou $\Omega$ ) est un ensemble compact.

Si $\Omega$ et $(\Gamma, a)$ sont en dualité, pour $z_{0} \in C$ et $\Omega_{1}=z_{0}+\Omega$ alors $\Omega_{1}$ est en dualité avec $(\Gamma, b)$ où $b(\xi)=a(\xi)-\operatorname{Im}\left(z_{0} \xi\right)$. De même $\Omega_{2}=e^{-i \theta} \Omega$ est en dualité avec $\left(\Gamma_{2}, b\right)$ où $\Gamma_{2}=e^{t \theta} \Gamma$ et $b(\xi)=a\left(e^{-i \theta} \xi\right)$.

Pour terminer ce chapitre voici quelques exemples.

i) ([11]). Soient $k_{1}, k_{2}$ et $b \in R$ tels que $k_{1} \leqq k_{2}$. Pour $\Gamma=$ $\{\xi \in C \mid \operatorname{Im} \xi>0\}$ il est clair que

$$
a(\xi)=\left\{\begin{array}{lll}
-b \operatorname{Im} \xi-k_{1} \operatorname{Re} \xi & \text { si } & \operatorname{Re} \xi \geqq 0 \\
-b \operatorname{Im} \xi-k_{2} \operatorname{Re} \xi & \text { si } & \operatorname{Re} \xi \leqq 0
\end{array}\right.
$$

est une fonction d'appui sur $\Gamma$. Dans ce cas $\Omega=\Omega(a, \Gamma)=[b,+\infty)+$ $i\left[k_{1}, k_{2}\right]$.

$$
\boldsymbol{\Omega}
$$

ii) Soient $\Gamma=\{\xi \in C \mid \operatorname{Re} \xi>0, \operatorname{Im} \xi>0\}$ et $a(\xi)=-(\operatorname{Im} \xi+\operatorname{Re} \xi)$. Alors $\Omega=\{z \in C \mid \operatorname{Re} z \geqq 1, \operatorname{Im} z \geqq 1\}$.

iii) ([5]) Soit $\Omega=\left\{z=x+i y \in C \mid e^{-x} \leqq 2 \cos y\right\}$. Alors

$\Gamma=\{\xi \in C \mid \operatorname{Im} \xi>0\}$ et $a(\xi)=\operatorname{Im} \xi \log \left(\frac{2 \operatorname{Im} \xi}{|\xi|}\right)-\operatorname{Re} \xi\left(\operatorname{Arg} \xi+\frac{\pi}{2}\right)$.

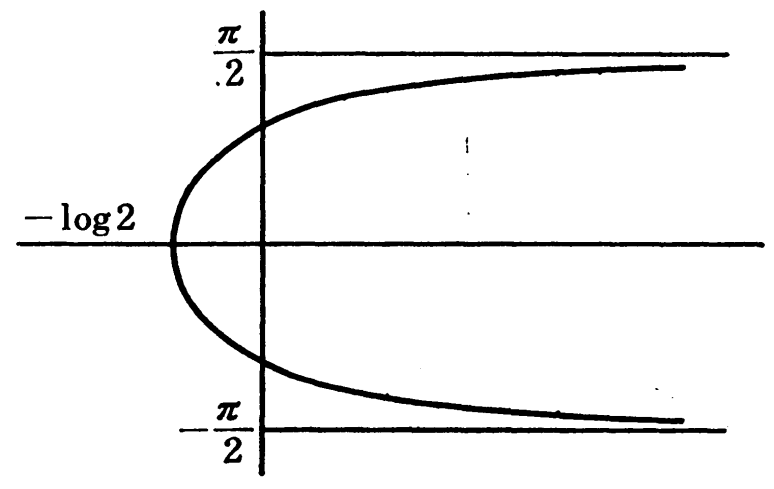

iv) Soit $\Gamma=\{\xi \in C \mid-\pi / 4<\operatorname{Arg} \xi<3 \pi / 4\}$. Il est clair que 


$$
a(\xi)=\left\{\begin{array}{ccc}
-\operatorname{Im} \xi & \text { si } & -\frac{\pi}{4}<\operatorname{Arg} \xi \leqq 0 \\
0 & \text { si } & 0 \leqq \operatorname{Arg} \xi \leqq \frac{\pi}{2} \\
-\operatorname{Re} \xi & \text { si } & \frac{\pi}{2} \leqq \operatorname{Arg} \xi<\frac{3 \pi}{4}
\end{array}\right.
$$

est une fonction d'appui sur $\Gamma$. Dans ce cas $\Omega$ est l'ensemble représenté par la figure ci-dessous.

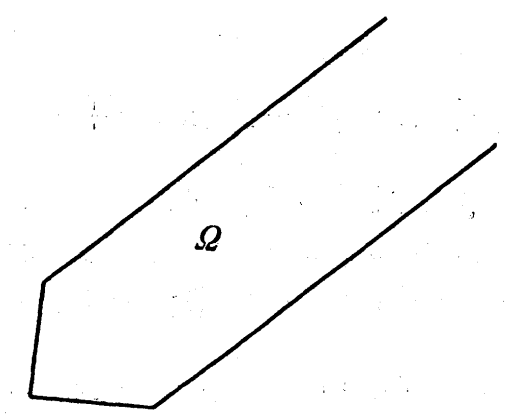

\section{Chapitre I}

FONCtionNelles ANALYTiques à PORTEUR NON BORN

Dans ce chapitre, $\Omega$ sera un ensemble convexe fermé non compact de $C$, distinct de $C$, en dualité avec $(\Gamma, a)$ où $\Gamma$ est un secteur ouvert convexe et $a$ une fonction d'appui sur $\Gamma$.

\section{§1.1. Espace $\mathscr{H}(\Omega)$.} de $\Gamma$.

On désigne par $\xi_{0}$ le point de $\operatorname{pr}(\Gamma)$ se trouvant sur la "bissectrice" Pour $\varepsilon>0$ et $\varepsilon^{\prime}>0$ soient $\mathscr{K}_{a_{,}^{\prime}}(\Omega)$ l'espace de Banach des $f \in \mathscr{H}\left(\dot{\Omega}_{\varepsilon}\right)$ telles que $\|f\|_{\varepsilon, \varepsilon^{\prime}}=\sup _{z \in} \dot{B}_{\varepsilon}\left|f(z) e^{-i e^{\prime} \xi_{0 z} z}\right|<+\infty$ et $\mathscr{H}_{0, \varepsilon, s^{\prime}}(\Omega)$ son sous-espace constitué des fonctions qui se prolongent par continuité à $\Omega_{c}$ et sont telles que $\lim _{\substack{z \in Q_{s} \\|z| \rightarrow \infty}}\left|f(z) e^{-i t^{\prime} \hat{s}_{0} z}\right|=0$.

LEMME 1.1.1. Il existe $K>0$ et $K_{1} \in R$ tels que, pour $w \in \Omega_{c} \quad(\varepsilon>0$ donné) on ait $\operatorname{Im}\left(\xi_{0} w\right) \geqq K|w|+K_{1}$.

Preuve. Elémentaire.

Proposition 1.1.2. Pour $\varepsilon>\varepsilon_{1}>0, \varepsilon^{\prime}>\varepsilon_{1}^{\prime}>0$ l'injection canonique 


$$
\pi_{i_{1}, \ell_{1}^{\prime}}^{\varepsilon, \varepsilon^{\prime}}: \mathscr{H}_{\varepsilon, \varepsilon^{\prime}}(\Omega) \longrightarrow \mathscr{H}_{\varepsilon_{1}, \varepsilon_{1}^{\prime}}(\Omega)
$$

est continue et compacte. Le système $\left(\mathscr{H}_{e, e^{\prime}}(\Omega), \pi_{\varepsilon_{1}, e_{1}^{\prime}}^{c, c_{1}^{\prime}}\right)$ est un système inductif d'espaces de Banach dont on notera $\mathscr{H}(\Omega)$ la limite inductive topologique qui est un espace du type D.F.S.

Pour $\alpha>\alpha_{1}>0, \alpha^{\prime}>\alpha_{1}^{\prime}>0$ l'injection canonique

$$
\theta_{\alpha_{1}, \alpha_{1}^{\prime}}^{\alpha, \alpha^{\prime}}: \mathscr{H}_{0, \alpha, \alpha^{\prime}}(\Omega) \longrightarrow \mathscr{K}_{0, \alpha_{1}, \alpha_{1}^{\prime}}(\Omega)
$$

est continue et compacte. Le système $\left(\mathscr{H}_{0, \alpha, \alpha^{\prime}}(\Omega), \theta_{\alpha_{1}, \alpha_{1}^{\prime}}^{\alpha, \alpha}\right)$ est un système inductif qui détermine aussi $\mathscr{H}(\Omega)$.

Preuve. Si $f \in \mathscr{K}_{\varepsilon, \varepsilon^{\prime}}(\Omega)$ l'inégalité

$$
\left|f(z) \exp \left(-i \varepsilon_{1}^{\prime} \xi_{0} z\right)\right| \leqq \exp \left[\left(\varepsilon^{\prime}-\varepsilon_{1}^{\prime}\right)\left(a\left(\xi_{0}\right)+\varepsilon_{1}\right)\right]\left|f(z) \exp \left(-i \varepsilon^{\prime} \xi_{0} z\right)\right|
$$

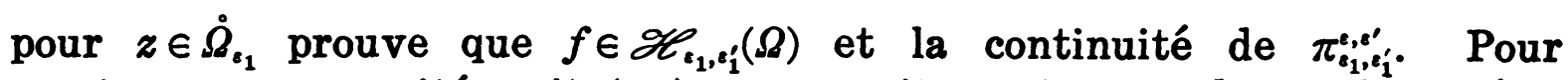
montrer sa compacité, soit $\left(m_{k}\right)_{k \geq 1}$ une suite croissante de nombres réels tendant vers $+\infty$ avec $k$ telle que $m_{1} \geqq-a\left(\xi_{0}\right)-\varepsilon_{1}$. L'ensemble

$$
K_{k}=\dot{\Omega}_{\varepsilon_{1}} \cap\left\{z \mid \operatorname{Im}\left(\xi_{0} z\right) \leqq m_{k}\right\}
$$

est d'adhérence compacte contenue dans $\dot{\Omega}_{\varepsilon}$ et $K_{k} \subset K_{k+1} \subset \dot{\Omega}_{\varepsilon_{1}}$ avec $\dot{\Omega}_{\varepsilon_{1}}=$ $\bigcup_{k \geq 1} K_{k}$. $z \in \dot{\Omega}_{\varepsilon_{1}} \backslash K_{k}$ implique que:

$$
\exp \left(\varepsilon_{1}^{\prime} \operatorname{Im} \xi_{0} z\right) \leqq \exp \left(\left(\varepsilon_{1}^{\prime}-\varepsilon^{\prime}\right) m_{k}\right) \exp \left(\varepsilon^{\prime} \operatorname{Im} \xi_{0} z\right) .
$$

Pour la seconde affirmation on remarque successivement que:


même sous-espace de $\mathscr{K}_{i_{1}, \varepsilon_{1}^{\prime}}(\Omega)$ (on notera $j_{i_{1}, \varepsilon_{1}^{\prime}}$ cette injection canonique) d'après 1.1.1.

b) $\theta_{\alpha_{1}, \alpha_{1}^{\prime}}^{\alpha, \alpha}=\pi_{\alpha_{1}, \alpha_{1}^{\prime}}^{\alpha, \alpha} \circ j_{\alpha, \alpha^{\prime}}$.

Ce qui assure que $\mathscr{H}(\Omega)$ est, algébriquement, égal à chacune des limites inductives déterminées par les deux systèmes inductifs considérés.

Les deux topologies d'espace D.F.S qu'on déduit sur $\mathscr{H}(\Omega)$ étant plus fines que la topologie séparée de la convergence simple, le théorème du graphe fermé prouve qu'elles sont identiques (cf. [4]). D'où la proposition.

\$1.2. Intégration sur le bord d'un ensemble convexe de $C$.

Une "route sans fin" $(\gamma,[a, b[)$ est une application

$$
\gamma:[a, b[\longrightarrow C \quad(-\infty<a<b \leqq+\infty)
$$

absolument continue telle que $\lim _{t \rightarrow b}|\gamma(t)|=+\infty$. On dit qu'une fonction 
continue au voisinage de $\gamma([a, b[)$ est intégrable le long de $\gamma$ si $t \rightarrow$ $f(\gamma(t)) \gamma^{\prime}(t)$ est intégrable sur $[a, b[$.

On conserve la notation usuelle:

$$
\int_{r} f(z) d z=\int_{[a, b[} f(\gamma(t)) \gamma^{\prime}(t) d t .
$$

Deux routes $\left(\gamma_{1},\left[a_{1}, b_{1}[)\right.\right.$ et $\left(\gamma_{2},\left[a_{2}, b_{2}[)\right.\right.$ seront dites équivalentes si $\gamma_{1}=$ $\gamma_{2} \circ \varphi$ où $\phi$ est une bijection absolument continue ainsi que son inverse de $\left[a_{1}, b_{1}\left[\right.\right.$ aur $\left[a_{2}, b_{2}\left[\right.\right.$ telle que $\varphi\left(a_{1}\right)=a_{2}$ et $\lim _{t \rightarrow b_{1}} \varphi(t)=b_{2}$.

Si $f$ est intégrable le long de $\gamma_{1}$ alors $f$ est aussi intégrable le long de $\gamma_{2}$ et on a

$$
\int_{r_{1}} f(z) d z=\int_{r_{2}} f(z) d z .
$$

On définit de même des routes sans fin du type $(\gamma] c, d]$,$) et (\gamma] e,, f[)$.

Proposition 1.2.1. Le bord $\partial F$ d'un convexe fermé non vide, non borné et distinct de $C$ est le support d'une route sans fin.

Preuve. Si $F$ n'est pas un demi-plan fermé, on peut supposer que $F \subset\{z \in C \mid \operatorname{Re} z \geqq 0\}$ la droite $\operatorname{Re} z=0$ étant une droite d'appui et que, pour tout $x \geqq 0$ :

$$
(x+i \boldsymbol{R}) \cap \boldsymbol{F}=x+i J_{x}
$$

où $J_{x}=[h(x), g(x)]$ est un intervalle compact non vide de $\boldsymbol{R}$ tel que $h(x)<g(x)$ pour $x>0$ si $F$ n'est pas une demi-droite. Les fonctions $g$ et $h$ ainsi définies sur $\boldsymbol{R}^{+}$sont convexe et concave respectivement donc absolument continue. D'où la proposition.

§1.3. Représentation intégrale de Cauchy pour $\mathscr{H}_{s, \varepsilon^{\prime}}(\Omega)$.

THEOR̀̀me 1.3.1. (Représentation intégrale de Cauchy). Pour $f \in$ $\mathscr{H}_{\varepsilon, \varepsilon^{\prime}}(\Omega)$ et $\varepsilon_{1}^{\prime}<\varepsilon^{\prime}, t_{1}<t<\varepsilon$ et $z \in \Omega_{t_{1}}$ on a

$$
f(z)=\frac{1}{2 i \pi} \int_{L_{t}} f(w) \frac{e^{i \varepsilon_{1}^{\prime} \varepsilon_{0}(z-w)}}{w-z} d w
$$

où $L_{t}$ est la route sans fin bord de $\Omega_{t}$.

Preuve. Pour $R$ assez grand l'intersection $\Delta_{R} \cap \Omega$ est un compact non vide avec $\Delta_{R}=\left\{w \mid \operatorname{Im} \xi_{0} w=R\right\}$.

Le théorème de Cauchy appliqué au circuit $L_{t, R}$ suggéré ci-dessous 


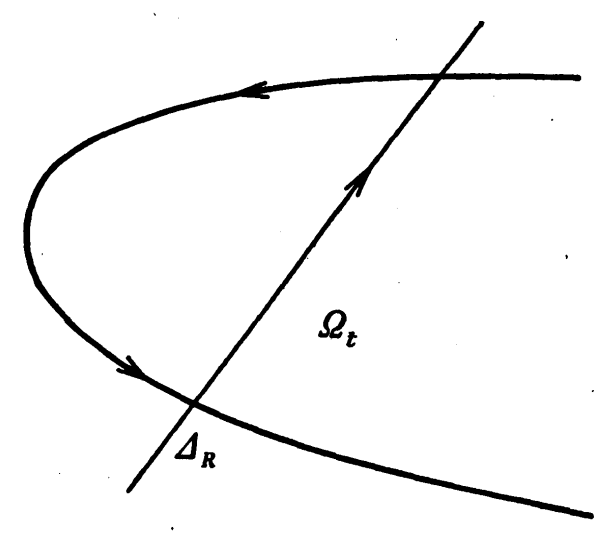

donne

$$
f(z)=\frac{1}{2 i \pi} \int_{\Sigma_{t, R}} f(w) \frac{e^{i t^{\prime} \varepsilon_{0}(z-w)}}{w-z} d w .
$$

On démontre classiquement que
a) $w \longrightarrow \frac{f(w) e^{i c_{1}^{\prime} \xi_{0}(z-w)}}{w-z}$

est intégrable le long de $L_{t}$ grâce à 1.1.1.

$$
\text { b) } \begin{aligned}
\lim _{R \rightarrow+\infty} \int_{L_{t, R \cap \Delta_{R}}} \frac{f(w) e^{i t_{1}^{\prime} \xi_{0}(z-w)}}{w-z} d w=0, \\
\lim _{R \rightarrow \infty} \frac{1}{2 i \pi} \int_{L_{t, R}} \frac{f(w) e^{i t_{1}^{\prime} \xi_{0}(z-w)}}{w-z} d w=\frac{1}{2 i \pi} \int_{L_{t}} \frac{f(w) e^{i t_{1}^{\prime} \xi_{0}(z-w)}}{w-z} d w .
\end{aligned}
$$

D'où le théorème.

'Proposition 1.3.2. La correspondance $f \rightarrow f^{\prime}$ est une application linéaire continue de $\mathscr{C}(\Omega)$ dans lui-même.

Preuve. Pour $0<\varepsilon_{1}^{\prime}<\varepsilon^{\prime}, 0<t_{1}<t<\varepsilon$ et $f \in \mathscr{K}_{\varepsilon, \varepsilon^{\prime}}(\Omega)$ on prouve comme précédemment la représentation $f^{\prime}(z)-i \varepsilon_{1}^{\prime} \xi_{0} f(z)=G(z)\left(z \in \Omega_{t_{1}}\right)$ avec

$$
G(z)=\frac{1}{2 i \pi} \int_{\Sigma_{t}} \frac{f(w) e^{i \varepsilon_{1}^{\prime} \varepsilon_{0}(z-w)}}{(w-z)^{2}} d w .
$$

Majorant en suite $|G(z)|$ en utilisant 1.1.1 on obtient une inégalité

$$
\|G\|_{t_{1}, e_{1}^{\prime}} \leqq C\|f\|_{e, \varepsilon^{\prime}} \quad(C \geqq 0) .
$$

Ce qui achève la démonstration.

En vue d'obtenir une transformation de Cauchy pour les éléments $\mu$ de $\mathscr{H}^{\prime}(\Omega)$ on remarque, pour $w \in \Omega^{\circ}$, la fonction: 


$$
z \longrightarrow \frac{e^{i \varepsilon^{\prime} \xi_{0}(z-w)}}{w-z}
$$

est un élément de $\mathscr{H}(\Omega)$, ce qui permet de considérer le nombre complexe

$$
\varphi_{\varepsilon^{\prime}}(w)=\frac{1}{2 i \pi}\left\langle\mu_{z}, \frac{e^{i \varepsilon^{\prime} \xi_{0}(z-w)}}{w-z}\right\rangle
$$

et on démontre la

Proposition 1.3.3. Soient $\mu \in \mathscr{H}^{\prime}(\Omega), f \in \mathscr{H}(\Omega), \varepsilon, \varepsilon^{\prime}, \varepsilon^{\prime \prime}$ et $t$ des réels $>0$ tels que $f \in \mathscr{H}_{\varepsilon^{\prime} \varepsilon^{\prime \prime}}(\Omega), t<\varepsilon$ et $\varepsilon^{\prime}<\varepsilon^{\prime \prime}$. On a

$$
\langle\mu, f\rangle=\int_{L_{t}} f(w) \varphi_{\varepsilon^{\prime}}(w) d w
$$

Preuve. Soient $0<\alpha<t_{1}<t<\varepsilon$ de telle sorte que $f \in \mathscr{H}_{0, \alpha, \varepsilon^{\prime}}(\Omega)$. La forme linéaire $\mu$ restreinte à $\mathscr{H}_{0, \alpha, \delta^{\prime}}(\Omega)$ se représente classiquement par une mesure bornée $d \nu$ sur $\Omega_{\alpha}$ pour laquelle

$$
\langle\mu, f\rangle=\int_{\Omega_{\alpha}} f(z) e^{-i s^{\prime} \varepsilon_{0} z} d \nu(z) \quad \text { (cf. [13]). }
$$

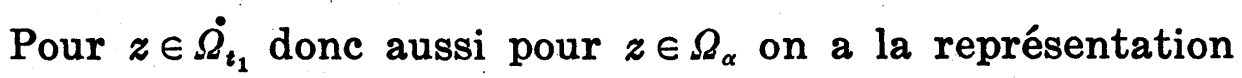

$$
f(z)=\frac{1}{2 i \pi} \int_{\Sigma_{t}} f(w) \frac{e^{i^{\prime} \xi_{0}(z-w)}}{w-z} d w
$$

D'où

$$
\langle\mu, f\rangle=\int_{\Omega_{\alpha}}\left(\int_{L_{t}} f(w) \frac{e^{-i \varepsilon^{\prime} \xi_{0}(z-w)}}{w-z} d w\right) d \nu(z)
$$

Comme $|w-z| \geqq t-t_{1}$ on voit qu'on peut conclure en utilisant le théorème de Fubini, la fonction $w \rightarrow\left|f(w) e^{-i \varepsilon^{\prime} \varepsilon_{0} w}\right|$ étant intégrable sur $L_{t}$ pour la mesure $|d w|$. D'où la proposition.

\section{§1.4. Espace $H_{\Omega}^{1}(C)$. Transformation de Cauchy.}

On se propose de définir un espace qui sera celui des transformées de Cauchy des éléments de $\mathscr{H}(\Omega)$. On suit la construction de [11].

Pour $\varepsilon^{\prime}>0$ soient $H_{\varepsilon^{\prime}}\left(\Omega^{\circ}\right)$ l'ensemble des fonctions holomorphes dans $\Omega^{\circ}$ telles que, pour tout $0<\varepsilon<r$

$$
\boldsymbol{q}_{r, \varepsilon}^{\varepsilon^{\prime}}(f)=\sup _{w \in S_{r} \mid \dot{w}_{\varepsilon}}\left|f(w) e^{i \varepsilon^{\prime} \xi_{0} w}\right|<+\infty
$$

et $H_{\varepsilon^{\prime}}(C)$ l'ensemble des fonctions entières telles que, pour tout $\varepsilon>0$ 


$$
p_{\varepsilon}^{\varepsilon^{\prime}}(f)=\sup _{w \in D_{\varepsilon}}\left|f(w) e^{i \varepsilon^{\prime} \varepsilon_{0} w}\right|<+\infty \text {. }
$$

La famille de semi-normes $\left(q_{r, \varepsilon}^{s^{\prime}}\right), 0<\varepsilon<r$, munit $H_{\varepsilon^{\prime}}\left(\Omega^{\circ}\right)$ d'une topologie d'espace de Fréchet. On désignera par $H_{\varepsilon^{\prime}, \Omega}^{1}(C)$ le quotient de $H_{\varepsilon^{\prime}}\left(\Omega^{\circ}\right)$ par son sous-espace $H_{\varepsilon^{\prime}}(C)$.

Pour $\varepsilon_{1}^{\prime}<\varepsilon^{\prime}$ l'espace $H_{\varepsilon_{1}^{\prime}}\left(\Omega^{c}\right)$ est un sous-ensemble de $H_{\varepsilon^{\prime}}\left(\Omega^{c}\right)$. En effet, pour $0<\varepsilon<r, w \in \Omega_{r} \mid \dot{\Omega}_{\varepsilon}$ et $f \in H_{\varepsilon_{1}^{\prime}}\left(\Omega^{\circ}\right)$

$$
|f(w)| \leqq q_{r, \varepsilon}^{\varepsilon_{1}^{\prime}}(f) e^{\varepsilon_{1}^{\prime} \operatorname{Im} \xi_{1}^{\prime} 0 w}=q_{r, \varepsilon}^{\varepsilon_{1}^{\prime}}(f) e^{\varepsilon^{\prime} \operatorname{Im} \xi_{0} w} e^{\left(\varepsilon^{\prime}-\varepsilon_{1}^{\prime}\right) \operatorname{Im} \xi_{0} w} .
$$

Il existe une constante $C>0$ telle que $e^{\left(\varepsilon_{1}^{\prime}-\varepsilon^{\prime}\right) \operatorname{Im} \varepsilon_{0} w} \leqq C$ pour tout $w \in \Omega_{r} \mid \dot{\Omega}_{e}$. Ceci résulte de la compacité de $\Omega_{r} \cap\left\{w \mid \operatorname{Im} \xi_{0} w \leqq 0\right\}$.

De même $H_{\varepsilon_{1}^{\prime}}(C)$ est contenu dans $H_{\varepsilon^{\prime}}(C)$ et l'injection canonique $H_{\varepsilon_{1}^{\prime}}\left(\Omega^{c}\right) \rightarrow H_{\varepsilon^{\prime}}\left(\Omega^{c}\right)$ induit par passage aux quotients, une application naturelle $J_{\varepsilon^{\prime}, \varepsilon_{1}^{\prime}}$ de $H_{\varepsilon_{1}^{\prime}, \Omega}^{1}(C)$ dans $H_{\varepsilon^{\prime}, \Omega}^{1}(C)$.

Proposition 1.4.1. 1) L'espace $H_{s^{\prime}}(C)$ est un sous-espace fermé de $H_{\varepsilon^{\prime}}\left(\Omega^{\circ}\right)$ et $H_{\varepsilon^{\prime}, \Omega}^{1}(C)$ est naturellement un espace de Fréchet.

2) Les applications $J_{\varepsilon^{\prime}, \varepsilon_{1}^{\prime}}$ sont injectives, linéaires et continues.

3) Le système $\left(H_{\varepsilon^{\prime}, o}^{1}(C), J_{\varepsilon^{\prime}, e_{1}^{\prime}}\right)$ est un système projectif.

PreUve. 1) Soit $\left(f_{n}\right)_{n \geqq 1}$ une suite d'éléments de $H_{\varepsilon^{\prime}}(C)$ qui converge dans l'espace $H_{a^{\prime}}\left(\Omega^{\circ}\right)$ vers un élément $f$.

Pour $0<\varepsilon<r$ fixés et $k \in N$ il existe un entier $n(k)$ tel que $n, p \geqq n(k)$ implique $q_{r, s}^{\varepsilon^{\prime}}\left(f_{n}-f_{p}\right) \leqq 1 / k$. On a aussi $p_{r}^{\varepsilon^{\prime}}\left(f_{n}-f_{p}\right) \leqq 1 / k$. Cela résulte du théorème classique de Phragmén-Lindelöf ([5]) qui donne:

Si $H$ est holomorphe au voisinage de $\Omega_{r}$ telle que $\sup _{z \in \rho_{r}}|H(z)| \leqq A$ et $|H(z)| \leqq M e^{N|z|}$ pour $z \in \Omega_{r}$ (où $A, M, N$ sont $>0$ ) alors sup $\operatorname{ses}_{r}|H(z)| \leqq A$.

Par suite il existe une fonction $f_{r}$ telle que la suite $\left(f_{n}\right)_{n \geq 1}$ converge uniformément vers $f_{r}$ sur tout compact de $\Omega_{r}$. La collection des $f_{r}(r>0)$ se recolle en une fonction entière $f_{0}$ qui prolonge $f$ et appartient à $H_{\varepsilon^{\prime}}(C)$. On vérifie que $n \geqq n(k)$ implique $p_{r}^{e^{\prime}}\left(f_{n}-f_{0}\right) \leqq 1 / k$. D'où 1$)$.

2) Le théorème de Phragmén-Lindelöf assure que $H_{\varepsilon^{\prime}}(C) \cap H_{\varepsilon_{1}^{\prime}}\left(\Omega^{\circ}\right) \subset$ $H_{\varepsilon_{1}^{\prime}}(C)$.

3) Elémentaire.

Notation 1.4.2. On note $H_{\Omega}^{1}(C)$ la limite projective $\lim _{8^{\prime}>0} H_{\varepsilon^{\prime}, \Omega}^{1}(C)$.

Theorìme 1.4.3. Soit $\mu \in \mathscr{H}^{\prime}(\Omega)$. La famille de fonctions $\left(\varphi_{\varepsilon^{\prime}}\right)_{\varepsilon^{\prime}>0}$, où

$$
\varphi_{\prime^{\prime}}(w)=\frac{1}{2 i \pi}\left\langle\mu_{x}, \frac{e^{i \varepsilon^{\prime} \xi_{0}(z-w)}}{w-z}\right\rangle \quad\left(w \in \Omega^{c}\right)
$$


définit un élément de $H_{\Omega}^{1}(C)$ qui sera noté $\mathscr{C}(\mu)$ et appelé la "transformée de Cauchy" de $\mu$. L'application $\mathscr{C}$ de $\mathscr{H}^{\prime}(\Omega)$ dans $H_{\Omega}^{1}(C)$ est linéaire et continue (transformation de Cauchy).

Preuve. L'holomorphie de $\varphi_{\varepsilon^{\prime}}$ (dont on sait déjà qu'elle est définie) dans $\Omega^{c}$ résulte de la représentation de $\mu$ par une mesure bornée convenable et des théorèmes de Morera et de Fubini.

La continuité de $\mu$ donne l'existence de $C_{\varepsilon, \varepsilon^{\prime}}>0$ telle que, pour $f \in$ $\mathscr{H}_{\varepsilon / 2, \varepsilon^{\prime}}(\Omega)$ on ait

$$
|\langle\mu, f\rangle| \leqq C_{\varepsilon, \varepsilon^{\prime}}\|f\|_{\varepsilon / 2, \varepsilon^{\prime}} \text {. }
$$

Or $w \in \Omega_{\varepsilon}^{c}$ implique que $f(z)=(1 / 2 i \pi) e^{i \varepsilon^{\prime} \xi_{0} z} /(w-z)$ est un élément de $\mathscr{H}_{\varepsilon / 2, \varepsilon^{\prime}}(\Omega)$ de norme majorée par $1 / \varepsilon \pi$. Par suite

$$
\left|\frac{1}{2 i \pi}\left\langle\mu_{z}, \frac{e^{i \varepsilon^{\prime} \varepsilon_{0} z}}{w-z}\right\rangle\right| \leqq \frac{C_{\varepsilon, \varepsilon^{\prime}}}{\varepsilon \pi} .
$$

Ainsi $\varphi_{\varepsilon^{\prime}} \in H_{\varepsilon^{\prime}}\left(\Omega^{\circ}\right)$.

Si maintenant on a $0<\varepsilon^{\prime}<\varepsilon_{1}^{\prime}$, la fonction $\varphi_{\varepsilon^{\prime}}-\varphi_{\varepsilon_{1}^{\prime}}$ est prolongeable en un élément de $H_{\varepsilon_{1}^{\prime}}(C)$. On considère

$$
G(\zeta)=\frac{e^{-i \varepsilon^{\prime} \xi_{0} \zeta}-e^{-i \varepsilon_{1}^{\prime} \xi_{0} \zeta}}{\zeta}
$$

qui est entière de $\zeta$.

Pour $\varepsilon>0, z \in \Omega_{2 \varepsilon}$ et $w \in \Omega_{\varepsilon}$ tels que $|w-z| \geqq \varepsilon$ on a l'inégalité

$$
\left|e^{i \xi_{0}\left(\varepsilon_{1}^{\prime}-\varepsilon^{\prime}\right)(z-w)}-1\right| \leqq 1+e^{\left(\varepsilon_{1}^{\prime}-\varepsilon^{\prime}\right)\left(a\left(\xi_{0}\right)+2 \varepsilon\right)} e^{\left(\varepsilon_{1}^{\prime}-\varepsilon^{\prime}\right) \operatorname{Im} \xi_{0} w}
$$

qui est donc aussi vraie pour tout $z \in \Omega_{2 \varepsilon}$ par le principe du maximum.

L'égalité

$$
e^{-i \varepsilon^{\prime} \xi_{0} z} G(w-z)=e^{-i \varepsilon^{\prime} \xi_{0} w}\left[\frac{e^{i \xi_{0}\left(\varepsilon_{1}^{\prime}-\varepsilon^{\prime}\right)(z-w)}-1}{z-w}\right]
$$

implique

$$
\|G(w-\cdot)\|_{\varepsilon, \varepsilon^{\prime}} \leqq \frac{e^{\varepsilon^{\prime} \operatorname{Im} \varepsilon_{0} w}}{\varepsilon}\left(1+e^{\left(\varepsilon_{1}^{\prime}-\varepsilon^{\prime}\right)\left(a\left(\xi_{0}\right)+2 \varepsilon\right)} e^{\left(\varepsilon_{1}^{\prime}-\varepsilon^{\prime}\right) \operatorname{Im} \xi_{0} w}\right)
$$

Ainsi, pour $w \in \Omega_{8}$ fixé, la fonction $z \rightarrow G(w-z)$ est un élément de $\mathscr{H}_{\varepsilon^{\prime}, 2 \varepsilon}(\Omega)$ et la fonction $H: w \rightarrow(1 / 2 i \pi)\left\langle\mu_{z}, G(w-z)\right\rangle$ existe et vérifie, pour des constantes convenables $A_{\varepsilon, \varepsilon^{\prime}, \varepsilon_{1}^{\prime}}$ et $B_{\varepsilon, \varepsilon^{\prime}, \varepsilon_{1}^{\prime}}$

$$
\left|\frac{1}{2 i \pi}\left\langle\mu_{z}, G(w-z)\right\rangle\right| \leqq A_{\varepsilon, \varepsilon^{\prime}, \varepsilon_{1}^{\varepsilon_{1}}} e^{\varepsilon^{\prime} \operatorname{Im} \xi_{0} w}+B_{\varepsilon, \varepsilon^{\prime}, \varepsilon_{1}^{\prime}} e^{\varepsilon_{1}^{\prime} \operatorname{Im} \xi_{0} w}
$$


et, bien sûr, prolonge $\varphi_{t^{\prime}}-\varphi_{t_{i}^{\prime}}$.

On constate que $H$ est holomorphe dans $\Omega_{\text {e }}$ comme précédemment et

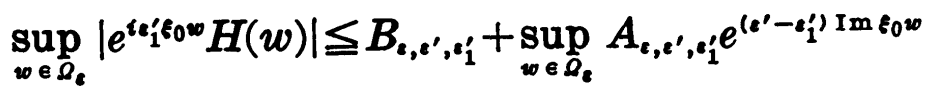

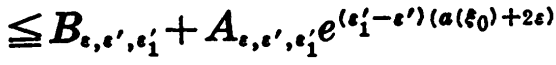

assure que $H=\varphi_{\varepsilon^{\prime}}-\varphi_{s_{1}^{\prime}}$ est élément de $H_{s_{1}^{\prime}}(C)$.

On notera $\bar{\varphi}_{\varepsilon^{\prime}}$ la classe de $\varphi_{\varepsilon^{\prime}}$ dans $H_{\varepsilon^{\prime}, \Omega}^{1}(C)$. Soit $\Psi$ l'élément de $\Pi_{\varepsilon^{\prime}>0} H_{\varepsilon^{\prime}, Q}^{1}(\boldsymbol{C})$ égal à $\left(\bar{\phi}_{\varepsilon^{\prime}}\right)_{\varepsilon^{\prime}>0}$. Puisque $\varphi_{\varepsilon^{\prime}}-\phi_{\varepsilon_{1}^{\prime}} \in H_{\varepsilon^{\prime}, Q}^{1}(C)$, on a $J_{t^{\prime}, \varepsilon_{1}^{\prime}}\left(\bar{\phi}_{\varepsilon^{\prime}}\right)=$ $\bar{\phi}_{\varepsilon^{\prime}}$. Ainsi $\Psi$ détermine un élément $\mathscr{C}(\mu)$ de l'espace $\lim _{\mathfrak{c}^{\prime}>0} H_{\varepsilon^{\prime}, Q}^{1}(\boldsymbol{C})$.

Enfin la continuité de l'application $\mathscr{C}$ résulte $\overleftarrow{\text { de }}$ la continuité des applications $\mu \rightarrow \varphi_{\iota^{\prime}}$ de $\mathscr{X}^{\prime}(\Omega)$ dans $H_{\mathbf{a}^{\prime}}\left(\Omega^{\circ}\right)$ qui provient elle-même de

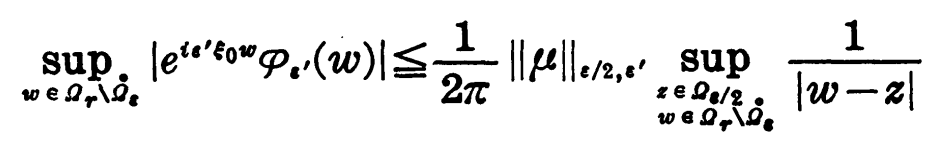

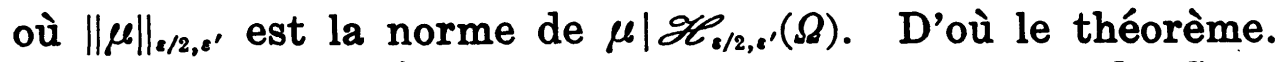

Nous allons démontrer que la transformation de Cauchy $\mathscr{C}$ est un isomorphisme topologique en explicitant sa réciproque.

Proposition 1.4.4. Soient $\Psi=\left(\bar{\phi}_{a^{\prime}}\right)_{a^{\prime}>0}$ un élément de $H_{\rho}^{1}(C)$ et $f$ un élément de $\mathscr{H}_{c, t_{1}^{\prime}}(\Omega)$. Pour $0<t<\varepsilon$ et $0<\varepsilon^{\prime}<\varepsilon_{1}^{\prime}$ l'intégrale

$$
I\left(t, \varphi_{\mathbf{c}^{\prime}}\right)(f)=\int_{\Sigma_{t}} \phi_{\mathrm{c}^{\prime}}(w) f(w) d w
$$

existe et est indépendante de $0<t<\varepsilon$, du représentant $\varphi_{\varepsilon^{\prime}}$ de $\bar{\varphi}_{\mathbf{t}^{\prime}}$ et de $0<\varepsilon^{\prime}<\varepsilon_{1}^{\prime}$. De plus $f \rightarrow I\left(t, \varphi_{\varepsilon^{\prime}}\right)(f)$ est un élément de $\mathscr{H}_{\varepsilon^{\prime}, \varepsilon^{\prime}}^{\prime}(\Omega)$.

Preuve. On a par hypothèse

$$
\begin{aligned}
& |f(w)| \leqq\|f\|_{\varepsilon, \varepsilon_{1}^{\prime}} \exp \left(-\varepsilon_{1}^{\prime} \operatorname{Im} \xi_{0} w\right) \quad\left(w \in \Omega_{\iota}\right) \\
& \left|\varphi_{\iota^{\prime}}(w)\right| \leqq q_{,, t}^{*} \exp \left(\varepsilon^{\prime} \operatorname{Im} \xi_{0} w\right) \quad\left(w \in \dot{\Omega}_{\iota} \mid \dot{\Omega}_{t}\right),
\end{aligned}
$$

ce qui assure l'existence de l'intégrale considérée.

Pour $0<t_{1}<t<\varepsilon$ le théorème de Cauchy assure que

$$
\int_{\tau_{R}^{t_{1} t_{1}}} \boldsymbol{Q}_{\boldsymbol{\prime}^{\prime}}(w) f(w) d w=0
$$

où $\gamma_{R}^{t_{i}, t_{1}}$ est le circuit suggéré ci-dessous: 


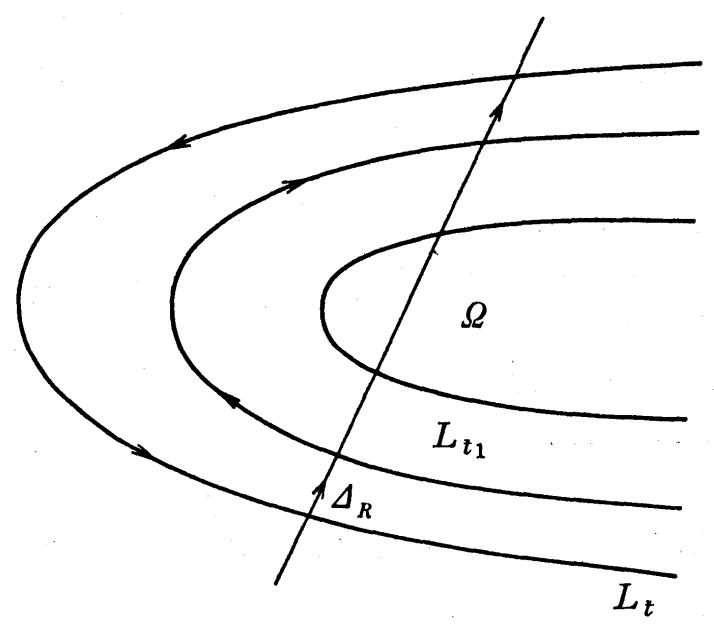

Les inégalités déjà citées impliquent que:

a) $\lim _{R \rightarrow+\infty} \int_{r_{R}^{t, t_{1} \Lambda_{R}}} \varphi_{\varepsilon^{\prime}}(w) f(w) d w=0$,

d'où l'indépendance de $I$ par rapport à $t$.

Les autres indépendances résultent de ce que $\varphi \in H_{\varepsilon^{\prime}}(\boldsymbol{C})$ pour $\varepsilon^{\prime}<\varepsilon_{1}^{\prime}$ implique

$$
\int_{L_{t}} \varphi(w) f(w) d w=0,
$$

b) la continuité de $I$.

D'où la proposition.

Cette proposition nous permet, suivant toujours en cela [11], d'introduire une application notée Int et appelée transformation intégrale

$$
\text { Int: } H_{\Omega}^{\prime}(C) \longrightarrow \mathscr{H}^{\prime}(\Omega)
$$

en posant

$$
\operatorname{Int}(\Psi)(f)=I\left(t, \varphi_{\varepsilon^{\prime}}\right)(f)
$$

pour $t$ et $\varphi_{\mathbf{a}^{\prime}}$ comme ci-dessus.

THEOREMe 1.4.5. L'application Int est l'application réciproque de l'application $\mathscr{C}$. Les deux sont des isomorphismes topologiques.

Preuve. L'égalité Int $\circ \mathscr{C}=\mathrm{id}_{\mathscr{E}^{\prime}(\Omega)}$ provient de 1.3.3. Prouvons donc que $\mathscr{C} \circ \operatorname{Int}=\mathrm{id}_{H_{Q}^{1}(C)}$.

Pour $\Psi=\left(\bar{\varphi}_{t^{\prime}}\right)_{\varepsilon^{\prime}>0}, \mu=\operatorname{Int}(\Psi)$ et $\mathscr{C}(\mu)=\left(\bar{\alpha}_{t^{\prime}}\right)_{\varepsilon^{\prime}>0}$ on a pour $f \in \mathscr{K}_{s, e_{1}^{\prime}}(\Omega)$ 
et $0<\varepsilon^{\prime}<\varepsilon_{1}^{\prime}$ :

$$
\int_{L_{t}}\left(\alpha_{\mathrm{s}^{\prime}}(w)-\varphi_{\mathrm{e}^{\prime}}(w)\right) f(w) d w=0
$$

par définition de $\mu$ et 1.3.3.

La fonction $\beta_{\varepsilon^{\prime}}=\alpha_{\varepsilon^{\prime}}-\varphi_{\varepsilon^{\prime}}$ se prolonge en un élément de $H_{\varepsilon^{\prime}}(C)$. La preuve est celle de [11].

La partie topologique résulte de la continuité de $\mathscr{C}(1.4 .3)$ et du théorème du graphe fermé.

\section{§1.5. Espace $\operatorname{Exp}(\Gamma, a)$ et transformation de Fourier-Borel.}

DEFINITION 1.5.1. Soient $\Gamma$ un secteur ouvert convexe non vide de $C$ de sommet l'origine et $a$ une fonction d'appui sur $\Gamma$. Le point $\xi_{0}$ étant le point de $\operatorname{pr}(\Gamma)$ se trouvant sur la bissectrice de $\Gamma$, soit $\Gamma_{\varepsilon^{\prime}}$ le secteur ouvert convexe $\varepsilon^{\prime} \xi_{0}+\Gamma$. Une fonction $f$ holomorphe dans $\Gamma$ sera dite de type exponentiel $a$ dans $\Gamma$ si, quels que soient $\varepsilon$ et $\varepsilon^{\prime}>0$, il existe $C_{\varepsilon, \varepsilon^{\prime}}(f) \geqq 0$ telle que

$$
|f(\zeta)| \leqq C_{\varepsilon, \varepsilon^{\prime}}(f) e^{a\left(\zeta-\varepsilon^{\prime} \xi_{0}\right)+\varepsilon|\zeta|} \quad\left(\zeta \in \Gamma_{\varepsilon^{\prime}}\right) .
$$

Proposition 1.5.2. Il existe une topologie naturelle d'espace $F . N$ sur l'espace $\operatorname{Exp}(\Gamma, a)$. soit

Preuve. Il suffit de vérifier les conditions $H . S_{1}$ et $H . S_{2}$. Pour cela

$$
M_{k}(\zeta)=e^{-a\left(\zeta-(1 / k) \xi_{0}\right)-(1 / k)|\zeta|} \quad\left(k \in N^{*}\right) .
$$

On a alors

$$
\operatorname{Exp}(\Gamma, a)=\lim _{k \geq 1} \mathscr{\mathscr { C }}\left(\Gamma_{1 / k}, M_{k}\right)
$$

(cf. 0.2.2 pour les notations).

Condition H.S. Elle provient de l'inégalité

$$
\left(\frac{M_{k}(\zeta)}{M_{k+1}(\zeta)}\right)^{2} \leqq \exp \left(\frac{2}{k(k+1)} a\left(\zeta_{0}\right)\right) \exp \left(\frac{-2|\zeta|}{k(k+1)}\right)
$$

qui assure l'intégrabilité de $M_{k} / M_{k+1}$ dans $\Gamma_{1 / k}$.

Condition H.S. Soit $d$ un nombre strictement positif et strictement plus petit que

$$
\inf \left(d\left(\Gamma_{1 / k}, \Gamma_{1 /(k+1)}^{c}\right), d\left(\frac{1}{k(k+1)} \xi_{0}, \Gamma^{c}\right)\right)
$$


où $d(A, B)$ désigne la distance des ensembles $A$ et $B$.

Pour tout $\zeta \in \Gamma_{1 / k}$ on pose $d_{\zeta}=d$ et on vérifie que

$$
\sup _{\substack{\zeta \in \Gamma_{1 / k} \\|\zeta-w| \leq d}} M_{k+1}^{-1}(w) M_{k}(\zeta)<+\infty
$$

(car $d_{\zeta}$ ne dépend pas de $\left.\zeta \in \Gamma_{1 / k}\right)$, en écrivant que $w-\xi_{0} /(k+1)=\left(\zeta-\xi_{0} / k\right)+$ $\left(w-\zeta+(1 / k-1 /(k+1)) \xi_{0}\right)$ et en remarquant que le choix de $d$ permet de considérer $a\left(w-\zeta+(1 /(k(k+1))) \xi_{0}\right)$.

Proposition 1.5.3. La correspondance $f \rightarrow f^{\prime}$ est une application linéaire continue de $\operatorname{Exp}(\Gamma, a)$ dans lui-même.

Preuve. Elle est élémentaire en utilisant le théorème de Cauchy.

Pour $\zeta \in \Gamma$ la fonction entière $e^{i z \zeta}$ définit un élément de $\mathscr{H}(\Omega)$. En effet si $\varepsilon^{\prime}>0$ est assez petit $\zeta-\varepsilon^{\prime} \xi_{0} \in \Gamma$ et

$$
\sup _{z \in D_{\varepsilon}}\left|e^{i z \zeta-i \varepsilon^{\prime} \varepsilon_{0} z}\right| \leqq e^{a\left(\zeta-\varepsilon^{\prime} \varepsilon_{0}\right)+\varepsilon\left|\zeta-\varepsilon^{\prime} \xi_{0}\right|} .
$$

On peut donc introduire la "transformation de Fourier-Borel" dans $\mathscr{H}^{\prime}(\Omega)$ en posant $\mathscr{F}(\mu)(\zeta)=\left\langle\mu_{z}, e^{i_{z} \zeta}\right\rangle \quad(\zeta \in \Gamma)$.

Proposition 1.5.4. Soit $\mu \in \mathscr{H}^{\prime}(\Omega)$, la fonction $\mathscr{F}(\mu)$ est élément de $\operatorname{Exp}(\Gamma, a) . \quad L a$ transformation $\mathscr{F}$ est linéaire et continue de $\mathscr{H}^{\prime}(\Omega)$ dans $\operatorname{Exp}(\Gamma, a)$.

PreUVE. L'holomorphie de $\mathscr{F}(\mu)$ se prouve en représentant $\mu$ par une mesure. La continuité de $\mu$ donne des inégalités

$$
|\mathscr{F}(\mu)(\zeta)| \leqq\|\mu\|_{\varepsilon, \varepsilon^{\prime}} e^{\varepsilon \varepsilon^{\prime}} e^{a\left(\zeta-\varepsilon^{\prime} \xi_{0}\right)+\varepsilon|\xi|}
$$

si $\zeta \in \Gamma_{\varepsilon^{\prime}}$. Ces inégalités assurent aussi la continuité de $\mathscr{F}$. D'où la proposition.

Nous allons introduire deux transformations dites respectivement de Laplace $(\mathscr{L})$ et de Polya $(\mathscr{P})$.

$$
\operatorname{Exp}(\Gamma, a) \underset{\mathscr{P}}{\stackrel{\mathscr{L}}{\rightleftarrows}} H_{\Omega}^{1}(C)
$$

réciproques l'une de l'autre vérifiant, de plus, $\mathscr{T}=\mathscr{P} \circ \mathscr{C}$ et $\mathscr{T}^{-1}=$ Int $\circ \mathscr{L}$.

Pour $f \in \operatorname{Exp}(\Gamma, a), \varepsilon^{\prime}>0$ et $\xi^{\prime} \in \operatorname{pr}(\Gamma)$ on considère la fonction de $w$ :

$$
F_{\varepsilon^{\prime}}\left(\xi^{\prime}, w\right)=\frac{1}{2 \pi} \int_{\varepsilon^{\prime} \xi_{0}+R^{+} \xi^{\prime}} f(\tau) e^{-i w \tau} d \tau
$$


qui est définie et holomorphe dans $H_{\xi^{\prime}}^{c}=\left\{w \in C \mid \operatorname{Im} w \xi^{\prime}<-a\left(\xi^{\prime}\right)\right\}$ (demi-plan s'appuyant sur $\Omega$ et ne contenant pas $\Omega$ ) comme on le constate classiquement.

De plus on vérifie que $F_{\iota^{\prime}}\left(\xi^{\prime}, w\right)=F_{\iota^{\prime}}\left(\xi^{\prime \prime}, w\right)$ pour $w \in H_{\xi^{\prime}}^{c} \cap H_{\xi^{\prime \prime}}^{c}$ grâce au théorème de Cauchy.

On dispose donc d'une famille de fonctions $\left(F_{a^{\prime}}\right)_{a^{\prime}>0}$ dans $\Omega^{c}=$ $\mathrm{U}_{\xi^{\prime} \mathrm{epr}(r)} H_{\xi^{\prime}}^{\circ}$ pour laquelle on a la

Proposition 1.5.5. La famille $\left(F_{t^{\prime}}\right)_{t^{\prime}>0}$ définit un élément de $H_{Q}^{1}(C)$ noté $\mathscr{L}(f)$ et appelé transformé de Laplace de $f$. L'application $\mathscr{L}$ (transformation de Laplace) est linéaire et continue.

PREUVE. Pour $\xi^{\prime} \in \operatorname{pr}(\Gamma)$ et $\varepsilon>0$ soit $H_{\xi^{\prime}, \varepsilon}^{\prime}=\left\{w \mid a\left(\xi^{\prime}\right)+\operatorname{Im} w \xi^{\prime}<-\varepsilon\right\}$ de telle sorte que $\Omega_{i}^{\circ}=\bigcup_{\xi^{\prime} \in \operatorname{pr}(\Gamma)} H_{\xi^{\prime}, \varepsilon}^{\prime}$.

Pour $w \in H_{\xi^{\prime}, \varepsilon}^{\prime}$ on a

$$
\left|e^{i \varepsilon^{\prime} \xi_{0} w} F_{\varepsilon^{\prime}}(w)\right| \leqq C_{s, c^{\prime}}(f) \int_{0}^{\infty} e^{-t(\varepsilon / 2)} d t .
$$

Ainsi $F_{\varepsilon^{\prime}} \in H_{\varepsilon^{\prime}}\left(\Omega^{\circ}\right)$. Maintenant, si $0<\varepsilon_{1}^{\prime}<\varepsilon^{\prime}$ on a l'expression

$$
F_{\varepsilon_{1}^{\prime}}(w)-F_{\varepsilon^{\prime}}(w)=\int_{\left[\varepsilon_{1}^{\prime} \varepsilon_{0}, \varepsilon^{\prime} \varepsilon_{0}\right]} f(\tau) e^{-i w \tau} d \tau
$$

( $\left[\varepsilon_{1}^{\prime} \xi_{0}, \varepsilon^{\prime} \xi_{0}\right]$ est le segment d'extrémités $\varepsilon_{1}^{\prime} \xi_{0}$ et $\left.\varepsilon^{\prime} \xi_{0}\right)$, qui se déduit des définitions et du théorème de Cauchy. Cela montre que $F_{a_{1}^{\prime}}-F_{a^{\prime}}$ se prolonge en un élément de $H_{z^{\prime}}(C)$. Par suite $\left(\bar{F}_{t^{\prime}}\right)_{\varepsilon^{\prime}}$ est un élément de $\boldsymbol{H}_{\Omega}^{1}(\boldsymbol{C})$.

La continuité de $\mathscr{L}$ résulte des calculs montrant l'appartenance $F_{\mathbf{s}^{\prime}} \in H_{\mathbf{a}^{\prime}}\left(\Omega^{\circ}\right)$.

Proposition 1.5.6. On a $\mathscr{L} \circ \mathscr{F}=\mathscr{C}$.

Preuve. Pour $\mu \in \mathscr{H}^{\prime}(\Omega), \quad \xi^{\prime} \in \operatorname{pr}(\Gamma), \varepsilon>0$ assez petit, $w \in H_{\xi^{\prime}}$ la fonction $(\tau, z) \rightarrow e^{\tau(z-w) \tau} \mathscr{\rho}_{\varepsilon^{\prime}}(z)$ est $(d|\tau| \times d|w|)$-intégrable sur $\left(\varepsilon^{\prime} \xi_{0}+R^{+} \xi^{\prime}\right) \times L_{c}$ pour $0<\varepsilon_{1}^{\prime}<\varepsilon^{\prime}$. Comme

$$
\begin{aligned}
(\mathscr{L} \circ \mathscr{F}(\mu))_{\varepsilon^{\prime}}(w) & =\frac{1}{2 \pi} \int_{{\mathcal{\iota ^ { \prime }}}^{\prime} \xi_{0}+R^{+} \xi^{\prime}} \mathscr{T}(\mu)(\tau) e^{-i w \tau} d \tau \\
& =\frac{1}{2 \pi} \int_{\varepsilon^{\prime} \xi_{0}+R^{+}+\xi^{\prime}}\left(\int_{L_{\varepsilon}} \varphi_{\varepsilon_{1}^{\prime}}(z) e^{i z \tau} d z\right) d \tau
\end{aligned}
$$

par 1.3.3. et

$$
(\mathscr{L} \circ \mathscr{F}(\mu))_{\varepsilon^{\prime}}(w)=\frac{1}{2 \pi} \int_{L_{\varepsilon^{\prime}}} \Phi_{\varepsilon_{1}^{\prime}}(z)\left(\int_{\varepsilon^{\prime} \xi_{0}+R^{+}+\xi^{\prime}} e^{z(z-w) \tau} d \tau\right) d z
$$


par le théorème de Fubini, on obtient

$$
((\mathscr{L} \circ \mathscr{T})(\mu))_{\varepsilon^{\prime}}=\mathscr{C}(\mu)_{\varepsilon^{\prime}}
$$

compte tenu de la définition de $\varphi_{\varepsilon_{1}^{\prime}}$ et de 1.3.3.

On introduit la "transformation de Polya" $\mathscr{P}$ en posant, pour $\Psi \doteq$ $\left(\bar{\Phi}_{\varepsilon^{\prime}}\right)_{a^{\prime}>0} \in H_{\Omega}^{1}(\boldsymbol{C})$

$$
\mathscr{P}(\Psi)(\zeta)=\int_{L_{\varepsilon}} e^{i w \zeta} \phi_{\boldsymbol{\varepsilon}^{\prime}}(w) d w .
$$

On constate que $\mathscr{P}(\Psi) \in \operatorname{Exp}(\Gamma, a)$ et que $\mathscr{P}^{\prime}: H_{\Omega}^{1}(C) \rightarrow \operatorname{Exp}(\Gamma, a)$ est linéaire et continue car $\mathscr{P}=\mathscr{F} \circ$ Int.

On se propose à présent de prouver que $\mathscr{P}$ et $\mathscr{L}$ sont réciproques l'une de l'autre. Voici tout d'abord un lemme dans l'énoncé duquel $\gamma_{c}$ désignera le chemin:

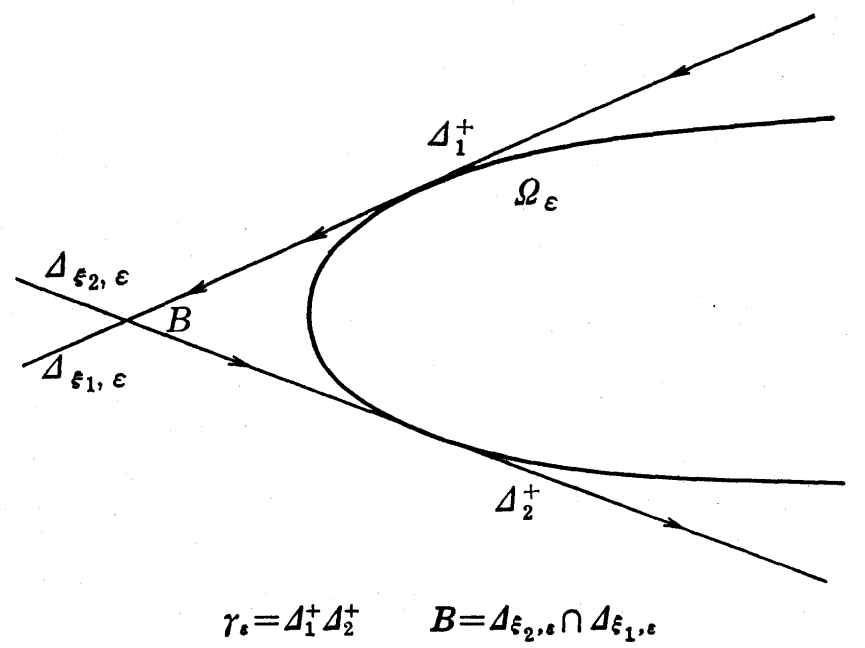

$\left(\xi_{1}, \xi_{2} \in \operatorname{pr} \Gamma\right.$ sont tels que $\xi_{0}$ soit intérieur au secteur $\Gamma_{1}$ limité par $\boldsymbol{R}^{+} \xi_{1}$ et $\left.\boldsymbol{R}^{+} \xi_{2}\right)$.

LEMME 1.5.7. Soit $\Psi \in H_{\partial}^{1}(\boldsymbol{C}), \Psi=\left(\bar{\phi}_{\varepsilon^{\prime}}\right)_{\varepsilon^{\prime}>0}$ tel que $\bar{\phi}_{\mathbf{s}^{\prime}}$ admette un représentant $\varphi_{\mathrm{t}^{\prime}}$, pour lequel $e^{i_{\mathbf{s}^{\prime}} \xi_{0} w} \varphi_{\mathrm{s}^{\prime}}(w)$ soit borné sur $\Omega_{\mathrm{s}}^{c}$ (un tel représentant existe, prendre $\left.\mathscr{C}(\operatorname{Int} \Psi)_{\varepsilon^{\prime}}\right)$. Si $\Gamma_{1}^{\prime}$ est un sous-secteur relativement compact de $\dot{\Gamma}_{1}$ et $\zeta \in \varepsilon^{\prime} \xi_{0}+\Gamma_{1}^{\prime}$ l'intégrale

$$
\int_{r^{\prime}} e^{i w \tau} \varphi_{\varepsilon^{\prime}}(w) d w
$$

converge pour $|\zeta|$ assez grand et vaut $\mathscr{P}(\Psi)(\zeta)$.

Preuve. De routine. 
THEORÈme 1.5.8. Les applications $\mathscr{L}$ et $\mathscr{P}$ sont des isomorphismes d'E.L.C réciproques l'un de l'autre.

Preuve. Soit $f \in \operatorname{Exp}(\Gamma, a)$ et $\Psi=\mathscr{L}(f)$. Pour $\lambda>\varepsilon^{\prime}$, on a

$$
\begin{aligned}
& \mathscr{P}(\Psi)\left(\lambda \xi_{0}\right)=\int_{r_{\varepsilon}} e^{i w \lambda \xi_{0}} F_{\varepsilon^{\prime}}(w) d w \\
& \quad=\frac{1}{2 \pi}\left[\int_{\Delta_{1}^{+}} e^{i w \lambda \varepsilon_{0}}\left(\int_{\epsilon^{\prime} \xi_{0}+R^{+} \xi_{1}} f(\tau) e^{-i w \tau} d \tau\right) d w+\int_{\Delta_{2}^{+}} e^{i w \lambda \xi_{0}}\left(\int_{\varepsilon^{\prime} \xi_{0}+R^{+} \xi_{2}} f(\tau) e^{-i w \tau} d \tau\right) d w\right] .
\end{aligned}
$$

On constate que le théorème de Fubini s'applique et on obtient

$$
\begin{aligned}
\mathscr{P}(\Psi)\left(\lambda \xi_{0}\right) & =\frac{1}{2 i \pi}\left[\int_{\varepsilon^{\prime} \xi_{0}+R^{+} \xi_{2}} f(\tau) \frac{e^{i B\left(\lambda \xi_{0}-\tau\right)}}{\tau-\lambda \xi_{0}} d \tau-\int_{\epsilon^{\prime} \xi_{0}+R^{+} \xi_{1}} f(\tau) \frac{e^{i B\left(\lambda \xi_{0}-\tau\right)}}{\tau-\lambda \xi_{0}} d \tau\right] \\
& =f\left(\lambda \xi_{0}\right)
\end{aligned}
$$

par le théorème de Cauchy.

On a donc $\mathscr{P} \circ \mathscr{L}=\operatorname{id}_{\mathrm{Exp}(r, a)}$ par prolongement analytique.

D'où le théorème.

Ce qui précède prouve aussi le

THEORÈm 1.5.9 ([15]). La transformation de Fourier-Borel est un isomorphisme d'E.L.C. de $\mathscr{H}^{\prime}(\Omega) \operatorname{sur} \operatorname{Exp}(\Gamma, a)$.

§1.6. Espaces $\mathscr{H}_{0}(\Omega), \operatorname{Exp}_{\bullet}(\Gamma, a)$ et isomorphismes de Fourier-Borel.

On peut introduire (cf. [15]) une notion (différente de celle venant d'être étudiée) de fonctions de type exponentiel dans un secteur $\Gamma$ relativement à une fonction d'appui $a$ de telle sorte que l'espace de ces fonctions apparaisse comme l'espace des sections d'un faisceau défini sur le compactifié radial de $C$ pour $a$ convenable. Ces fonctions sont aussi les transformées de Fourier-Borel des éléments du dual d'une limite inductive d'espaces de fonctions holomorphes définies dans des voisinages "coniques" de $\Omega$. Ces espaces, qui vont être décrits ci-dessous, sont justiciables de théorèmes analogues à ceux énoncés précédemment pour $\mathscr{H}(\Omega), \operatorname{Exp}(\Gamma, a)$ et $H_{\Omega}^{1}(C)$. Ces théorèmes seront énoncés sans démonstration celles-ci étant en tous points semblables à celles déjà-vues.

Soit $\left(\Gamma_{k}\right)_{k \geq 1}$ une exhaustion de $\Gamma$ (cf. 0.3). L'espace $\mathscr{H}_{0}(\Omega)$ est l'espace du type D.F.S

$$
\mathscr{H}_{0}(\Omega)=\lim _{k \geq 1} \mathscr{H}\left(\dot{\Omega}_{k, 0}, \exp \left(\frac{1}{k}|z|\right)\right)
$$

- où $\Omega_{k, o}$ est l'ensemble $\Omega\left(\Gamma_{k}, a(z)+(1 / k)|z|\right)$. 
On choisit $\Gamma_{1}$ de telle sorte que $\xi_{0} \in \Gamma_{1}$. On vérifie qu'algébriquement et topologiquement on a

$$
\mathscr{H}_{0}(\Omega)=\lim _{k \geq 1} \mathscr{H}\left(\dot{\Omega}_{k, o}, \exp \left(\frac{-i \xi_{0} z}{k K}\right)\right)
$$

où $K$ est un réel $>0$ tel que $\operatorname{Im} \xi_{0} w \geqq K|w|+K_{1}$ pour tout $w \in \Omega_{1,0}$ (conformément au lemme 1.1.1). De même

$$
\mathscr{H}_{0}(\Omega)=\lim _{k \geq 1} \mathscr{H}_{0}\left(\Omega_{k, c}, \exp \left(\frac{-i \xi_{0} z}{k K}\right)\right)
$$

où les fonctions $f$ de $\mathscr{H}_{0}\left(\Omega_{k, e}, \exp \left(-i \xi_{0} z / k K\right)\right)$ sont de plus continues sur $\Omega_{k, c}$ et sont telles que

$$
\lim _{\substack{z \in \Omega_{k} \\|z| \rightarrow \infty}}\left|f(z) \exp \left(\frac{-i \xi_{0} z}{k K}\right)\right|=0 .
$$

Pour $\varepsilon>0, \Omega_{k, \varepsilon, c}$ l'e-voisinage de $\Omega_{k, c}$ et $L_{k, \varepsilon}$ la route sans fin $\partial \Omega_{k, \varepsilon, c}$, on a le

Théonème 1.6.1. (Représentation intégrale de Cauchy). Pour $f \in$ $\mathscr{H}\left(\dot{\Omega}_{k, o}, \exp \left(-i \xi_{0} z / k K\right)\right), 0<\varepsilon<1 /(k(k+1))$ et $\varepsilon^{\prime}<\varepsilon$ on $a$

$$
f(z)=\frac{1}{2 i \pi} \int_{\Sigma_{k+1, \varepsilon}} f(w) \frac{e^{i \varepsilon_{0}(z-w) /((k+1) K)}}{w-z} d w \quad\left(z \in \Omega_{k+1, \varepsilon^{\prime}, c}\right) .
$$

REMARQUE. Si $w \in Q_{k+1,0}$ la fonction

$$
z \longrightarrow \frac{e^{i \xi_{0} z /((k+1) K)}}{w-z}
$$

est élément de $\mathscr{H}\left(\dot{\Omega}_{k+1, c}, \exp \left(-i \xi_{0} z /((k+1) K)\right)\right)$, si $\mu \in \mathscr{H}_{0}^{\prime}(\Omega)$ on définit pour $w \notin \Omega_{k+1,0}$

$$
\varphi_{k+1}(w)=\frac{1}{2 i \pi}\left\langle\mu_{z}, \frac{e^{i \varepsilon_{0}(z-w) /((k+1) K)}}{w-z}\right\rangle .
$$

Proposition 1.6.2. Pour $f \in \mathscr{H}\left(\dot{\Omega}_{k, o}, \exp \left(-i \xi_{0} z / k K\right)\right), \mu \in \mathscr{H}_{0}^{\prime}(\Omega)$ et $0<\varepsilon<1 /(k(k+1))$ on $a$

$$
\langle\mu, f\rangle=\int_{\Sigma_{k+1, \varepsilon}} f(w) \varphi_{k+1}(w) d w .
$$

Pour $k \geqq 2$, soit $H_{\Omega, k, c}$ l'espace des fonctions holomorphes $h$ dans $\Omega_{k, c}^{c}$ telles que pour tout $0<\varepsilon<1-1 / k$ 


$$
q_{k, \varepsilon}(h)=\sup _{z \in \Omega_{1, \ell} Q_{k, \ell, e}}\left|h(w) \exp \left(\frac{i \xi_{0} w}{k K}\right)\right| \text {. }
$$

Cet espace muni de ces normes auxquelles on adjoint celles de la convergence uniforme sur tout compact de $\Omega_{k}^{c}$ est un espace de Fréchet. Nous noterons $H_{k, o}(C)$ le sous-espace (fermé) de $H_{0, k, 0}$ constitué des fonctions entières $f$ telles que $\sup _{w \in \Omega_{1, \varepsilon}}\left|f(w) \exp \left(i w \xi_{0} / k K\right)\right|<+\infty$ et nous noterons $H_{k, 0}^{1}$ le quotient $H_{Q, k, o} / H_{k, o}(C)$ qui est aussi un espace de Fréchet. On a des applications naturelles $\varphi_{k+1, k}: H_{k+1,0}^{1} \rightarrow H_{k, 0}^{1}$ injectives et le système $\left(H_{k, 0}^{1}, \varphi_{k+1, k}\right)_{k \geq 2}$ est projectif et détermine un espace de Fréchet $H_{\Omega, c}^{1}=\lim _{\leftarrow \geq 2} H_{k, 0}^{1}$. Nous noterons $\dot{\varphi}_{k}$ la classe de $\varphi_{k}$ dans $H_{k, 0}^{1}$.

THEOREME 1.6.3. Soit $\mu \in \mathscr{H}_{0}^{\prime}(\Omega)$. La suite $\left(\dot{\varphi}_{k}\right)_{k \geq 2}$ est un élément de $H_{\Omega, o}^{1}$, noté $\mathscr{C}(\mu)$ appelé transformé de Cauchy de $\mu$. L'application $\mathscr{C}$ (transformation de Cauchy) est linéaire continue de $\mathscr{C}_{0}^{\prime}(\Omega)$ dans $H_{\Omega, \bullet}^{1}$.

Proposition 1.6.4. Soient $\psi=\left(\psi_{k}\right)_{k \geq 2}$ un élément de $H_{\Omega, o}^{1}, f \in \mathscr{H}_{0}(\Omega)$, $k$ tel que $f \in \mathscr{H}\left(\dot{\Omega}_{k, 0}, \exp \left(-i \xi_{0} z / k K\right)\right)$ et $0<\varepsilon<1 /(k(k+1))$. L'intégrale

$$
I_{\varepsilon, k}=\int_{L_{k+1, \ell}} \psi_{k+1}(z) f(z) d z
$$

est indépendante de $\varepsilon$ et de $k$ et définit un élément de $\mathscr{H}_{0}^{\prime}(\Omega)$ noté Int $(\psi)$. L'application Int est linéaire continue.

THEORÈme 1.6.5. Les applications Int et $\mathscr{C}$ sont réciproques l'une de l'autre.

L'espace $\operatorname{Exp}_{c}(\Gamma, a)$ est l'espace des fonctions holomorphes dans $\Gamma$ telles que, pour tout $\Gamma^{\prime} \subset \subset \Gamma$, tout $\varepsilon>0, \delta>0$ on ait

$$
\sup _{\substack{z \in \Gamma^{\prime} \\|z|>\delta}}|f(z) \exp (-a(z)-\varepsilon|z|)|<+\infty
$$

Désignons par $\Gamma(k)$ l'ensemble $\Gamma_{k} \cap\{z|| z \mid>1 / k\}$. L'espace $\operatorname{Exp}_{o}(\Gamma, a)$ sera, topologiquement

$$
\operatorname{Exp}_{c}(\Gamma, a)=\lim _{k \geq 1} \mathscr{C}\left(\Gamma(k), \exp \left(-a(z)-\frac{1}{k}|z|\right)\right) .
$$

C'est un espace du type F.N. On vérifie que

$$
\operatorname{Exp}_{\mathrm{o}}(\Gamma, a)=\underset{\leftarrow}{\lim } \mathscr{\mathscr { C }}\left(\Gamma^{\prime}(\dot{k}), \exp \left(-a(z)-\frac{1}{k}|z|\right)\right),
$$

où $\Gamma^{\prime}(k)=\Gamma_{k} \cap\{z|| z \mid>1 / k K\}$. 
Si $\xi \in \Gamma$ alors $z \rightarrow e^{i z \xi}$ est élément de $\mathscr{H}_{0}(\Omega)$ et, pour $\mu \in \mathscr{H}_{0}^{\prime}(\Omega)$ on définit la transformée de Fourier-Borel de $\mu$ par

$$
\mathscr{F} \mu(\xi)=\left\langle\mu_{z}, e^{i z \xi}\right\rangle \text {. }
$$

Proposition 1.6.6. Soit $\mu \in \mathscr{H}_{0}^{\prime}(\Omega)$. La fonction $\mathscr{F}(\mu)$ est élément de $\operatorname{Exp}_{\mathrm{o}}(\Gamma, \mathrm{a})$ et la transformation de Fourier-Borel $\mathscr{F}$ est linéaire continue de $\mathscr{Z}_{0}^{\prime}(\Omega)$ dans $\operatorname{Exp}_{\mathrm{o}}(\Gamma, a)$.

Pour $f \in \operatorname{Exp}_{o}(\Gamma, a)$ on pose

$$
F_{k}\left(\xi^{\prime}, w\right)=\frac{1}{2 \pi} \int_{R+\xi^{\prime}} f(\tau) e^{-i w \tau} d \tau \quad\left(\xi^{\prime} \in \Gamma_{k}\right) .
$$

Proposition 1.6.7. La collection $\left(F_{k}\left(\xi^{\prime}, \cdot\right)\right)_{\xi^{\prime} \in r_{k}}$ détermine, par recollement, une fonction $F_{k}$ holomorphe dans $\Omega_{k, 0}^{\circ}$. La fonction $F_{k} \in H_{\Omega, k, 0} \quad$ La famille $\left(F_{k}\right)_{k \geq 2}$ définit un élément $\mathscr{L}(f)$ de $H_{Q, 0}^{1}$ appelé transformée de Laplace de $f$. La transformation de Laplace $f \rightarrow \mathscr{L}(f)$ est linéaire continue de $\operatorname{Exp}_{c}(\Gamma, a)$ dans $H_{a, 0}^{1}$ et on a $\mathscr{L} \circ \mathscr{T}=\mathscr{C}$.

Si on pose $\mathscr{P}=\mathscr{T} \circ \mathscr{C}$ (transformation de "Polya") on a $\mathscr{P} \circ \mathscr{L}=$ $\operatorname{Id}_{\mathrm{Exp}_{o}(\Gamma, a)}$.

THEOREMe 1.6.8. La transformation de Fourier-Borel est un isomorphisme d'E.L.C entre $\mathscr{H}_{0}^{\prime}(\Omega)$ et $\operatorname{Exp}_{0}(\Gamma, a)$.

Remarque. Comme on peut s'y attendre les espaces $\operatorname{Exp}(\Gamma, a)$ et $\operatorname{Exp}_{c}(\Gamma, a)$ (resp. $\mathscr{H}(\Omega)$ et $\mathscr{C}_{0}(\Omega)$ ) sont assez différents même lorsque $a$ est borné sur pr $\Gamma$. Nous allons en fournir un exemple.

Soit $\Omega=[0,+\infty)+i[1 / 4,3 / 4]$, il est clair que $f(z)=e^{-z} / \sin z$ est élément de $\mathscr{H}(\Omega)$, mais n'est pas élément de $\mathscr{H}_{0}(\Omega)$ contrairement à ce qui est annoncé en [14].

\section{Bibliographie}

[1] C. A. Berenstein et B. A. Taylor, A new look at interpolation theory for entire functions of one variable, Advances in Math., 33 (1979), 109-143.

[2] C. A. Berenstein et D. Struppa, Interpolation problems in cones, Preprint, Univ. Orsay, 1981.

[3] R. P. BoAs, Entire Functions, Academic Press, 1954.

[4] A. Grothendigck, Espaces Vectoriels Topologiques, Publicaçao de Sociedade de Matematica de Sao Paulo 3 a Ediçao, 1964.

[5] E. Hille, Analytic Function Theory, vol. II, Blaisdell Publ. Company, 1962.

[6] T. KAWAY, On the theory of Fourier hyperfunctions and its application to partial differential equations with constant coefficients, J. Fac. Sci. Univ. Tokyo, Sect. IA 17 (1970), 467-483. 
[7] B. Malgrange, Rapport sur les théorèmes d'indice de Boutet de Monvel et Kashiwara. Prépubl. Institut Fourier, 1982.

[8] A. MÉrIL, Fonctionnelles analytiques à porteur non borné et applications, Thèse 3ème cycle, Bordeaux I, 1981.

[9] A. Meril, Analytic functionals with unbounded carriers and mean periodic functions, a paraitre au Trans. Amer. Math. Soc..

[10] A. MÉriL, Problèmes d'interpolation dans quelques espaces de fonctions non entières, preprint.

[11] M. МовIмото, Analytic functionals with non compact carriers, Tokyo J. Math., Vol. 1, $\mathrm{n}^{\circ} 1$ (1978), 77-103.

[12] M. Morimoto et P. Sargos, Transformation des founctionnelles analytiques à porteurs non compacts, Tokyo J. Math., vol. 4, $\mathrm{n}^{\circ} 2$ (1981), 457-492.

[13] J. W. de ROEVER, Fourier transform of holomorphic functions and application to Newton interpolation series I, Publ. Math. Centrum, Amsterdam, 1974.

[14] J. W. de ROEVER, Fourier transform of holomorphic functions and application to Newton interpolation series II, T.W. 148 Math. Centrum, Amsterdam, 1975.

[15] J. W. de RoEver, Complex Fourier transformation and analytic functionals with unbounded carriers, Math. Center. Trac. 89, Amsterdam, 1978.

Address actuelle:

UNIVERSITE DE BORDEAUX I

U.E.R. DE MATHEMATIQUES ET INFORMatiquE

LABORATOIRE ASSOCIE AU C.N.R.S. N ${ }^{\circ} 226$

351, CoUrs de LA Liberation

33405 Talence Cedex, France 